\title{
A two-index generalization of conformable operators with potential applications in engineering and physics
}

\author{
E. Reyes-Luis \\ Departamento de Física y Matemáticas, Universidad Iberoamericana, \\ Ciudad de México, México. \\ e-mail: rceduardo1l@gmail.com; \\ Orcid: 0000-0002-2586-3123 \\ G. Fernández-Anaya \\ Departamento de Física y Matemáticas, Universidad Iberoamericana, \\ Ciudad de México, México. \\ e-mai: guillermo.fernandez@ibero.mx; \\ Orcid: 0000-0002-2396-5046 \\ J. Chávez-Carlos \\ Instituto de Ciencias Físicas, Universidad Nacional Autónoma de México, \\ Av. Universidad s/ $n$ Col. Chamilpa, 62210 Cuernavaca, Morelos, México. \\ e-mail: jchavez@icf.unam.mx; \\ Orcid: 0000-0002-5223-5931

\section{Diago-Cisneros} \\ Facultad de Física. Universidad de La Habana, La Habana, Cuba. \\ Departamento de Física y Matemáticas, Universidad Iberoamericana, Ciudad de México, México. \\ e-mail: leovildo.diago@ibero.mx; \\ Orcid: 0000-0003-0569-0162 \\ R. Muñoz-Vega \\ Universidad Autónoma de la Ciudad de México, \\ Fray Servando Teresa de Mier 92, Col. Centro, Alcaldía Cuauhtémoc, CDMX, México 06080 \\ e-mail: rodrigo.munoz@uacm.edu.mx; \\ ORCID 0000-0002-4755-6722
}

Received 9 November 2020; accepted 24 December 2020

We developed a somewhat novel fractional-order calculus workbench as a certain generalization of Khalil's conformable derivative. Although every integer-order derivate can naturally be consistent with fully physical-sense problem's quotation, this is not the standard scenario of the non-integer-order derivatives, even aiming physics systems' modeling, solely. We revisited a particular case of the generalized conformable fractional derivative and derived a differential operator, whose properties overcome those of the integer-order derivatives, though preserving its clue advantages. Worthwhile noting that the two-fractional indexes differential operator we are dealing with departs from the singlefractional index framework, which typifies the generalized conformable fractional derivative. This distinction leads to proper mathematical tools, useful in generalizing widely accepted results, with potential applications to fundamental Physics within fractional order calculus. The latter seems to be especially appropriate for exercising the Sturm-Liouville eigenvalue problem, as well as the Euler-Lagrange equation, and to clarify several operator algebra matters.

Keywords: Conformable operators; algebraic methods; quantum operators; sturm Liouville operator.

DOI: https://doi.org/10.31349/RevMexFis.67.429

\section{Introduction}

Derivatives of non-integer order have been discussed for a long time. In 1695 L'Hôpital asked Leibniz what meaning could be ascribed to $D^{n} f$ if n were a fraction. Since that time, fractional calculus has drawn the attention of many mathematicians. Most of them have used an integral form to define the fractional derivative; the two most popular ones are the Riemann-Liouville definition and the Caputo definition. However, these definitions did not inherit most of the proper- ties of the classic integer-order derivative, such as the product rule or the chain rule. In 2014 Khalil et al. [1] introduced the definition of the conformable fractional derivative, analogous to the limit definition of the standard integer-order derivative. This definition was later generalized by Katugampola [2], whose work was later addressed by Anderson et al. [3], who used Katugampola's results to explore its properties and its potential application in quantum mechanics, see also [4-8]. In 2017, Al-Refai and Abdeljawad [9] suggested a generaliza- 
tion of the well-known Sturm-Liouville eigenvalue problem using the conformable derivative. In 2018 Zhao et al. [10] introduced a set of Maxwell's equations using the Generalized Conformable Fractional Derivative (GCFD), which obeys the classical properties of the integer-order derivative. Several works have recently appeared on conformal derivatives, e.g., [14-19]. Clearly showing that it is a field of great growth, despite its recent creation. Some applications of these conformal derivatives to optical solitons solutions are in $[20,21]$.

We generalize the conformable derivative introduced by Khalil et al. [1] by exploring the properties of a fractional derivative operator derived from the GCFD derivative. Unlike the rest of the fractional derivatives, the order of our operator is determined by two fractional indexes and two positive functions, which can be arranged to recover the integer order derivative. The two fractional indexes and the two functions give the derivative greater freedom and more complex dynamics than single-index and single-function derivatives; furthermore, being a conformable derivative, it is a local derivative just like in the integer-order case. In this way, our proposed GCFD is similar to the Gateaux derivative [10] but definitively is not equal. We present a differential operator in which the order of the derivative now depends on the two fractional indexes $\alpha$ and $\beta$, preserving almost all the properties of integer-order derivatives, as it is often the case with conformable local derivatives. We construct operators with potential application in quantum mechanics, generalizing some results obtained by Anderson and Ulness [3]. We generalize some well-known results, such as the EulerLagrange method [11] and the Sturm-Liouville eigenvalue problem [12] by replacing the integer-order derivative operators with our operator, following a similar procedure as Abdeljawad et al. [9] and we show some examples.

In the first section, we present the definition of the operator, its main properties, such as the product rule and the chain rule, as well as some examples. In the next section, we further explore its properties, we introduce an inverse operator, and then we explore its commutator and anti-commutator properties, as well as some results with applications in quantum mechanics. In the last section, we generalize some results obtained in the second section, namely the Sturm-Liouville operator. We consider the Sturm-Liouville eigenvalue problem using the differential operator introduced in the first section. We also generalize some results, such as the Lagrange Identity and the Euler-Lagrange equation.

\section{Preliminaries}

Let $\psi(u, \alpha, \beta)$ be a fractional conformable function and $\alpha, \beta \in(0,1]$. The " $\alpha \beta$ derivative" of $\mathrm{f}: \mathbb{R}^{+} \longrightarrow \mathbb{R}$ at $x$ is defined as:

$$
D_{\psi}^{\alpha, \beta}(f)(x)=\lim _{\epsilon \rightarrow 0} \frac{f(x+\epsilon \psi(x, \alpha, \beta))-f(x)}{\epsilon} .
$$

The restrictions of $\psi(u, \alpha, \beta)$ are:

$$
\begin{cases}\psi(x, 1,1)=1 & x \in \mathbb{R}^{+} \\ \psi(x, \alpha, \beta) \neq 0 & x \in \mathbb{R}^{+} \\ \psi\left(., \alpha_{1}, \beta\right) \neq \psi\left(., \alpha_{2}, \beta\right) & \alpha_{1} \neq \alpha_{2} \\ \psi\left(., \alpha, \beta_{1}\right) \neq \psi\left(., \alpha, \beta_{2}\right) & \beta_{1} \neq \beta_{2}\end{cases}
$$

where $\alpha_{i}$ and $\beta_{i} \in(0,1]$, for $i=1,2,3 \ldots$

Let $f:[0, \infty] \longrightarrow, \mathbb{R}$ and $\mathrm{x}>0$ also let $h(x)$ and $k(x)$ be continuous functions, where $h(x)>0$ and $k(x)>0$. Then the " $\alpha, \beta h k l$ conformable fractional derivative" of $f$ of order $\alpha, \beta$ is defined by:

$$
\mathrm{D}_{\mathrm{h}, \mathrm{k}, \mathrm{l}}^{\alpha, \beta}(f)(x)=\lim _{\epsilon \rightarrow 0} \frac{f\left(x+h^{\beta}(x) e_{l}^{\epsilon k^{1-\alpha}(x)}\right)-f(x)}{\epsilon},
$$

where

$$
e_{l}^{\epsilon k^{1-\alpha}(x)}=\sum_{j=1}^{l} \frac{\epsilon^{j} k^{(1-\alpha) j}(x)}{j !},
$$

for $\mathrm{x}>0, \alpha, \beta \in(0,1]$. If the limit exists, we say that $f(x)$ is $\gamma$-differentiable.

Recently M.N. Alam and Xin Li [13] solved the complex fractional Schrödinger equation using the conformable derivative introduced by Khalil et al. [1]; our fractional operator generalizes the operator introduced by Khalil.

If we set $h(x)=1, \beta=1, k(x)=x$ and $l=1$ then we recover the conformable fractional derivative definition from Khalil et al.:

$$
D_{1, t, 1}^{\alpha, 1}(f)(x)=\lim _{\epsilon \rightarrow 0} \frac{f\left(x+\epsilon x^{1-\alpha}\right)-f(x)}{\epsilon} .
$$

If we set $h(x)=x, \beta=1, k(x)=x^{\frac{\alpha}{\alpha-1}}$ and $l \rightarrow \infty$ then we recover the fractional derivative of order $\alpha$ definition from Katugampola:

$$
D_{x, x^{\frac{\alpha}{\alpha-1}}, \infty}^{\alpha, 1}(f)(x)=\lim _{\epsilon \rightarrow 0} \frac{f\left(x e^{\epsilon x^{-\alpha}(x)}\right)-f(x)}{\epsilon}
$$

\section{Properties of the differential operator}

Let $\alpha \in(0,1]$ and $f, g$ be $\gamma$-differentiable at a point $x>0$. Using Eq. (2) and Eq. (3), it can be easily proved that:

1. $\mathrm{D}_{\mathrm{h}, \mathrm{k}, \mathrm{l}}^{\alpha, \beta}(a f+b g)=a \mathrm{D}_{\mathrm{h}, \mathrm{k}, \mathrm{l}}^{\alpha, \beta}(f)+b \mathrm{D}_{\mathrm{h}, \mathrm{k}, \mathrm{l}}^{\alpha, \beta}(g), a, b \in \mathbb{R}$.

2. $\mathrm{D}_{\mathrm{h}, \mathrm{k}, \mathrm{l}}^{\alpha, \beta}\left(x^{n}\right)=n x^{n-1} h^{\beta}(x) k^{1-\alpha}(x), n \in \mathbb{R}$.

3. $\mathrm{D}_{\mathrm{h}, \mathrm{k}, \mathrm{l}}^{\alpha, \beta}(c)=0$, for all constant functions.

4. $\mathrm{D}_{\mathrm{h}, \mathrm{k}, \mathrm{l}}^{\alpha, \beta}(f g)=g(x) \mathrm{D}_{\mathrm{h}, \mathrm{k}, \mathrm{l}}^{\alpha, \beta}(f)+f(x) \mathrm{D}_{\mathrm{h}, \mathrm{k}, \mathrm{l}}^{\alpha, \beta}(g)$.

5. $\mathrm{D}_{\mathrm{h}, \mathrm{k}, 1}^{\alpha, \beta}\left(\frac{f}{g}\right)=\frac{g(x) \mathrm{D}_{\mathrm{h}, \mathrm{k}, 1}^{\alpha, \beta}(f)-f(x) \mathrm{D}_{\mathrm{h}, \mathrm{k}, 1}^{\alpha, \beta}(g)}{g^{2}(x)}$.

6. $\mathrm{D}_{\mathrm{h}, \mathrm{k}, \mathrm{l}}^{\alpha, \beta}(f \circ g)=\frac{d f}{d x}(g(x)) \mathrm{D}_{\mathrm{h}, \mathrm{k}, \mathrm{l}}^{\alpha, \beta}(g)(x)$. 
7. $\mathrm{D}_{\mathrm{h}, \mathrm{k}, 1}^{\alpha, \beta}(f)=h^{\beta}(x) k^{1-\alpha}(x) \frac{d f}{d x}$.

8. $\mathrm{D}_{\mathrm{h}, \mathrm{k}, \mathrm{l}}^{\alpha, \beta} f\left(y_{1}(x), \ldots, y_{N}(x)\right)=\frac{\partial f}{\partial y_{1}} \mathrm{D}_{\mathrm{h}, \mathrm{k}, \mathrm{l}}^{\alpha, \beta}\left(y_{1}\right)+\ldots+$ $\frac{\partial f}{\partial y_{N}} \mathrm{D}_{\mathrm{h}, \mathrm{k}, \mathrm{l}}^{\alpha, \beta}\left(y_{N}\right)$.

\section{The $\alpha \beta h k l$ conformable fractional derivate and its relationship with second-order lin- ear defferential equations}

We now further explore the properties of our differential operator Eq. (2) and develop some new operators from this one, such as the inverse operator, the commutator and the anti-commutator as well as a self-adjoint variant of the anticommutator. We will later use some of this results when solving the Sturm-Liouville eigenvalue problem, such as the integration by parts formula.

\subsection{The $\mathrm{D}_{\mathrm{h}, \mathrm{k}, \mathrm{l}}^{\alpha, \beta}$ operator}

We begin by exploring the properties of the $\mathrm{D}_{\mathrm{h}, \mathrm{k}, \mathrm{l}}^{\alpha, \beta}$ operator.

$$
\mathrm{D}_{\mathrm{h}, \mathrm{k}, \mathrm{l}}^{\alpha, \beta}[y(x)]=h^{\beta}(x) k^{1-\alpha}(x) \frac{d y(x)}{d x},
$$

where $d / d x$ is the integer-order derivative operator. So the $\mathrm{D}_{\mathrm{h}, \mathrm{k}, \mathrm{l}}^{\alpha, \beta}$ operator is

$$
\mathrm{D}_{\mathrm{h}, \mathrm{k}, \mathrm{l}}^{\alpha, \beta}=h^{\beta}(x) k^{1-\alpha}(x) \frac{d}{d x} .
$$

Next, we considered the iterated operator $\mathrm{D}_{\mathrm{h}, \mathrm{k}, \mathrm{l}}^{\alpha, \beta} \mathrm{D}_{\mathrm{h}, \mathrm{k}, \mathrm{l}}^{\rho, \sigma}$

$$
\begin{aligned}
\mathrm{D}_{\mathrm{h}, \mathrm{k}, \mathrm{l}}^{\alpha, \beta}\left[\mathrm{D}_{\mathrm{h}, \mathrm{k}, \mathrm{l}}^{\rho, \sigma}[y]\right] & =h^{\beta}(x) k^{1-\alpha}(x) \frac{d}{d x}\left[h^{\sigma}(x) k^{1-\rho}(x) y^{\prime}\right] \\
& =h^{\beta}(x) k^{1-\alpha}(x)\left(\sigma h^{\sigma-1}(x) h^{\prime}(x)\right. \\
& \times k^{1-\rho}(x) y^{\prime}+(1-\rho) h^{\sigma}(x) k^{-\rho}(x) \\
& \left.\times k^{\prime}(x) y^{\prime}+h^{\sigma}(x) k^{1-\rho}(x) y^{\prime \prime}\right) .
\end{aligned}
$$

If we let $\gamma_{1}=\alpha+\beta$ and $\gamma_{2}=\beta+\sigma$, then:

$$
\begin{aligned}
& =h^{\gamma_{2}}(x) k^{2-\gamma_{1}}(x) y^{\prime \prime}+\left(\sigma h^{\gamma_{2}-1}(x) h^{\prime}(x)\right. \\
& \left.\times k^{2-\gamma_{1}}(x)+(1-\rho) h^{\gamma_{2}}(x) k^{1-\gamma_{1}}(x) \times k^{\prime}(x)\right) y^{\prime} .
\end{aligned}
$$

\subsection{The operator $\mathrm{I}_{\mathrm{h}, \mathrm{k}, \mathrm{l}}^{\alpha, \beta}$}

We define the inverse operator of $\mathrm{D}_{\mathrm{h}, \mathrm{k}, \mathrm{l}}^{\alpha, \beta}$ as

$$
\begin{aligned}
\left(\mathrm{D}_{\mathrm{h}, \mathrm{k}, \mathrm{l}}^{\alpha, \beta}\right)^{-1} \equiv \mathrm{I}_{\mathrm{h}, \mathrm{k}, \mathrm{l}}^{\alpha, \beta} & =\int^{x}(.) k^{\alpha-1}(t) h^{-\beta}(t) \mathrm{d} t \\
& =\int^{x}(.) d \gamma(t),
\end{aligned}
$$

where (.) is a place holder for the function to be operated upon. yields

Applying the anti-derivative operator $\mathrm{I}_{\mathrm{h}, \mathrm{k}, 1}^{\alpha, \beta}$ to $\mathrm{D}_{\mathrm{h}, \mathrm{k}, 1}^{\alpha, \beta}$

$$
\mathrm{I}_{\mathrm{h}, \mathrm{k}, \mathrm{l}}^{\alpha, \beta}\left[\mathrm{D}_{\mathrm{h}, \mathrm{k}, \mathrm{l}}^{\alpha, \beta}[y]\right]=\int^{x} y(t) \mathrm{d} \gamma(t)=y(x),
$$

where one takes $y$ to vanish at the lower limit. Now applying the $\mathrm{D}_{\mathrm{h}, \mathrm{k}, \mathrm{l}}^{\alpha, \beta}$ operator to $\mathrm{I}_{\mathrm{h}, \mathrm{k}, \mathrm{l}}^{\alpha, \beta}$ yields

$$
\begin{aligned}
\mathrm{D}_{\mathrm{h}, \mathrm{k}, \mathrm{l}}^{\alpha, \beta}\left[\mathrm{I}_{\mathrm{h}, \mathrm{k}, 1}^{\alpha, \beta}[y]\right] & =\mathrm{D}_{\mathrm{h}, \mathrm{k}, \mathrm{l}}^{\alpha, \beta}\left[\int^{x} y(t) \mathrm{d} \gamma(t)\right]=h^{\beta}(x) k^{1-\alpha}(x) \frac{d}{d x} \\
& \times\left[\int^{x} y(t) \mathrm{d} \gamma(t)\right]=y(x)
\end{aligned}
$$

However, in the mixed case $\mathrm{D}_{\mathrm{h}, \mathrm{k}, \mathrm{l}}^{\rho, \sigma} \mathrm{I}_{\mathrm{h}, \mathrm{k}, \mathrm{l}}^{\alpha, \beta}$ yields:

$$
\begin{aligned}
\mathrm{D}_{\mathrm{h}, \mathrm{k}, \mathrm{l}}^{\rho, \sigma}\left[\mathrm{I}_{\mathrm{h}, \mathrm{k}, \mathrm{l}}^{\alpha, \beta}[y]\right] & =\mathrm{D}_{\mathrm{h}, \mathrm{k}, \mathrm{l}}^{\rho, \sigma}\left[\int^{x} y(t) \mathrm{d} \gamma(t)\right] \\
& =h^{\sigma}(x) k^{1-\rho}(x) \frac{d}{d x}\left[\int^{x} y(t) \mathrm{d} \gamma(t)\right],
\end{aligned}
$$

let $\delta_{1}=\alpha-\rho$ and $\delta_{2}=\sigma-\beta$

$$
=h^{\delta_{2}}(x) k^{\delta_{1}}(x) y(x) .
$$

Similarly for $\mathrm{I}_{\mathrm{h}, \mathrm{k}, \mathrm{l}}^{\alpha, \beta} \mathrm{D}_{\mathrm{h}, \mathrm{k}, \mathrm{l}}^{\rho, \sigma}$ we have

$$
\begin{aligned}
\mathrm{I}_{\mathrm{h}, \mathrm{k}, 1}^{\alpha, \beta}\left[\mathrm{D}_{\mathrm{h}, \mathrm{k}, 1}^{\rho, \sigma}[y]\right] & =\int^{x} k^{\alpha-1}(t) h^{-\beta}(t) h^{\sigma}(t) k^{1-\rho}(t) y^{\prime} \mathrm{d} t \\
= & \int k^{\delta_{1}}(t) h^{\delta_{2}}(t) y^{\prime}(t) \mathrm{d} t \\
& =h^{\delta_{2}}(x) k^{\delta_{1}}(x) y(x) \\
& -\delta_{1} \int^{x} k^{\delta_{1}-1}(t) k^{\prime}(t) h^{\delta_{2}}(t) y(t) \mathrm{d} t
\end{aligned}
$$

$$
\begin{aligned}
& -\delta_{2} \int^{x} h^{\delta_{2}-1}(t) h^{\prime}(t) k^{\delta_{1}}(t) y(t) \mathrm{d} t \\
& =h^{\delta_{2}}(x) k^{\delta_{1}}(x) y(x) \\
& -\delta_{1} I_{h, k, l}^{\delta_{1}, \delta_{2}}\left[h^{2 \delta_{2}}(x) k^{\prime}(x) y(x)\right] \\
& -\delta_{2} I_{h, k, l}^{\delta_{1}, \delta_{2}}\left[h^{2 \delta_{2}-1}(x) h^{\prime}(x) k(x) y(x)\right] .
\end{aligned}
$$

Using the definition for the anti-derivative operator, we can also derive a formula for integration by parts.

Theorem 4.1 (Integration by parts) 
Let $f, g:[a, b] \rightarrow \mathbb{R}$ be two functions such that $f g$ is differentiable, then:

$$
\begin{gathered}
\int_{a}^{b} f(x) \mathrm{D}_{\mathrm{h}, \mathrm{k}, \mathrm{l}}^{\alpha, \beta} g(x) \mathrm{d} \gamma(x)=\left.f(x) g(x)\right|_{a} ^{b} \\
\quad-\int_{a}^{b} g(x) \mathrm{D}_{\mathrm{h}, \mathrm{k}, \mathrm{l}}^{\alpha, \beta} f(x) \mathrm{d} \gamma(x) .
\end{gathered}
$$

proof

$$
\begin{aligned}
& \int_{a}^{b} f(x) \mathrm{D}_{\mathrm{h}, \mathrm{k}, \mathrm{l}}^{\alpha, \beta} g(x) \mathrm{d} \gamma(x)=\int_{a}^{b} f(x) h^{\beta}(x) k^{1-\alpha}(x) g^{\prime}(x) \\
& \quad \times \mathrm{d} \gamma(x)=\left.f(x) g(x)\right|_{a} ^{b}-\int_{a}^{b} g(x) f^{\prime}(x) \mathrm{d} x=\left.f(x) g(x)\right|_{a} ^{b} \\
& \quad-\int_{a}^{b} g(x) h^{\beta}(x) k^{1-\alpha}(x) f^{\prime}(x) h^{-\beta}(x) k^{\alpha-1}(x) \mathrm{d} x \\
& =\left.f(x) g(x)\right|_{a} ^{b}-\int_{a}^{b} g(x) \mathrm{D}_{\mathrm{h}, \mathrm{k}, \mathrm{l}}^{\alpha, \beta} f(x) \mathrm{d} \gamma(x) .
\end{aligned}
$$

\subsection{Parity in the $D_{h, k, l}^{\alpha, \beta}$ operator}

We can neglect the requirement that $\mathrm{t}>0$ and consider the parity of $\mathrm{D}_{\mathrm{h}, \mathrm{k}, \mathrm{l}}^{\alpha, \beta}$ by applying the operator $\widehat{P}$ on Eq. (7):

$$
\begin{aligned}
\widehat{P} \mathrm{D}_{\mathrm{h}, \mathrm{k}, \mathrm{l}}^{\alpha, \beta} & =\widehat{P}\left[h^{\beta} k^{1-\alpha}(x) \frac{d}{d x}\right] \\
& =h^{\beta}(-x) k^{1-\alpha}(-x)\left(-\frac{d}{d x}\right) .
\end{aligned}
$$

We may consider two cases for powers of $h(x)$ and $k(x)$ of the form:

$$
\nu_{1}=\frac{1}{2 \mathrm{n}+1}
$$

and

$$
\nu_{2}=\frac{2 \mathrm{n}}{2 \mathrm{n}+1}
$$

where $n \in \mathbb{Z}_{\geq 0}$

So, the action of $\widehat{P}$ becomes:

$$
\widehat{P} D_{h, k, l}^{1-\nu_{i}, \nu_{j}}=h^{\nu_{j}}(-x) k^{\nu_{i}}(-x)\left(-\frac{d}{d x}\right),
$$

where $i, j=1,2$. Taking into account the parity of $h(x)$ and $k(x)$, we explore the different cases for $\widehat{P}$ :

4.3.1. $\nu_{1}$

1.

$$
\widehat{P} D_{h, k, l}^{1-\nu_{1}, \nu_{1}}=h^{\frac{1}{2 \mathrm{n}+1}}(-x) k^{\frac{1}{2 \mathrm{n}+1}}(-x)\left(-\frac{d}{d x}\right),
$$

(1) $h(x)$ even and $k(x)$ even or $h(x)$ odd and $k(x)$ odd

$$
\widehat{P} D_{h, k, l}^{1-\nu_{1}, \nu_{1}}=e^{i \pi} D_{h, k, l}^{1-\nu_{1}, \nu_{1}},
$$

(2) $h(x)$ even and $k(x)$ odd or $h(x)$ odd and $k(x)$ even

$$
\widehat{P} D_{h, k, l}^{1-\nu_{1}, \nu_{1}}=D_{h, k, l}^{1-\nu_{1}, \nu_{1}} .
$$

\subsection{2. $\quad \nu_{2}$}

1.

$$
\widehat{P} D_{h, k, l}^{1-\nu_{2}, \nu_{2}}=h^{\frac{2 \mathrm{n}}{2 \mathrm{n}+1}}(-x) k^{\frac{2 \mathrm{n}}{2 \mathrm{n}+1}}(-x)\left(-\frac{d}{d x}\right) .
$$

(1) $h(x)$ even and $k(x)$ even or $h(x)$ odd and $k(x)$ odd or $h(x)$ even and $k(x)$ odd or $h(x)$ odd and $k(x)$ even

$$
\widehat{P} D_{h, k, l}^{1-\nu_{2}, \nu_{2}}=e^{i \pi} D_{h, k, l}^{1-\nu_{2}, \nu_{2}} .
$$

\subsection{3. $\nu_{1}$ and $\nu_{2}$}

1.

$$
\widehat{P} D_{h, k, l}^{1-\nu_{2}, \nu_{1}}=h^{\frac{1}{2 \mathrm{n}+1}}(-x) k^{\frac{2 \mathrm{n}}{2 \mathrm{n}+1}}(-x)\left(-\frac{d}{d x}\right),
$$

(1) $h(x)$ even and $k(x)$ even or $h(x)$ even and $k(x)$ odd

$$
\widehat{P} D_{h, k, l}^{1-\nu_{2}, \nu_{1}}=e^{i \pi} D_{h, k, l}^{1-\nu_{2}, \nu_{1}},
$$

(2) $h(x)$ odd and $k(x)$ even or $h(x)$ odd and $k(x)$ odd

$$
\widehat{P} D_{h, k, l}^{1-\nu_{2}, \nu_{1}}=D_{h, k, l}^{1-\nu_{2}, \nu_{1}} .
$$

2.

$$
\widehat{P} D_{h, k, l}^{1-\nu_{1}, \nu_{2}}=h^{\frac{2 \mathrm{n}}{2 \mathrm{n}+1}}(-x) k^{\frac{1}{2 \mathrm{n}+1}}(-x)\left(-\frac{d}{d x}\right),
$$

(1) $h(x)$ even and $k(x)$ even or $h(x)$ odd and $k(x)$ even

$$
\widehat{P} D_{h, k, l}^{1-\nu_{1}, \nu_{2}}=e^{i \pi} D_{h, k, l}^{1-\nu_{1}, \nu_{2}},
$$

(2) $h(x)$ even and $k(x)$ odd or $h(x)$ odd and $k(x)$ odd

$$
\widehat{P} D_{h, k, l}^{1-\nu_{1}, \nu_{2}}=D_{h, k, l}^{1-\nu_{1}, \nu_{2}} .
$$

\subsection{First order differential equation}

We consider a differential equation for the $\mathrm{D}_{\mathrm{h}, \mathrm{k}, \mathrm{l}}^{\alpha, \beta}$ operator:

$$
\mathrm{D}_{\mathrm{h}, \mathrm{k}, \mathrm{l}}^{\alpha, \beta}[y(x)]+\lambda y(x)=0 .
$$

Using Eq. (6) and solving for $y(x)$ :

$$
\begin{array}{r}
h^{\beta}(x) k^{1-\alpha}(x) y^{\prime}(x)+\lambda y(x)=0 \\
y^{\prime}(x)+\lambda h^{-\beta}(x) k^{\alpha-1}(x) y(x)=0 \\
\frac{d}{d x}\left[e^{\lambda \int^{x} h^{-\beta}(t) k^{\alpha-1}(t) \mathrm{d} t} y(x)\right]=0 \\
y(x)=c e^{-\lambda \int^{x} h^{-\beta}(t) k^{\alpha-1}(t) \mathrm{d} t} .
\end{array}
$$




\subsection{The commutator with the $D_{h, k, l}^{\alpha, \beta}$ derivative}

The operators $\mathrm{D}_{\mathrm{h}, \mathrm{k}, \mathrm{l}}^{\alpha, \beta}$ and $\mathrm{D}_{\mathrm{h}, \mathrm{k}, \mathrm{l}}^{\rho, \sigma}$ do not commute, and unlike integer-order derivatives $\mathrm{D}_{\mathrm{h}, \mathrm{k}, \mathrm{l}}^{\alpha, \beta} \mathrm{D}_{\mathrm{h}, \mathrm{k}, \mathrm{l}}^{\rho, \sigma} \neq D_{k, l}^{\alpha+\rho, \beta+\sigma}$; however, it is interesting to explore the commutator properties of these operators.

$$
\left[\mathrm{D}_{\mathrm{h}, \mathrm{k}, \mathrm{l}}^{\alpha, \beta}, \mathrm{D}_{\mathrm{h}, \mathrm{k}, 1}^{\rho, \sigma}\right] y=\mathrm{D}_{\mathrm{h}, \mathrm{k}, \mathrm{l}}^{\alpha, \beta}\left[\mathrm{D}_{\mathrm{h}, \mathrm{k}, \mathrm{l}}^{\rho, \sigma}[y]\right]-\mathrm{D}_{\mathrm{h}, \mathrm{k}, \mathrm{l}}^{\rho, \sigma}\left[\mathrm{D}_{\mathrm{h}, \mathrm{k}, \mathrm{l}}^{\alpha, \beta}[y]\right] .
$$

From Eq. (8):

$$
\begin{aligned}
& =\left((\sigma-\beta) h^{\beta+\sigma-1}(x) h^{\prime}(x) k^{2-\alpha-\rho}(x)\right. \\
& \left.+(\alpha-\rho) h^{\beta+\sigma}(x) k^{1-\alpha-\rho}(x) k^{\prime}(x)\right) y^{\prime} \\
& =\left(\delta_{2} h^{\gamma_{2}-1}(x) h^{\prime}(x) k^{2-\gamma_{1}}(x)\right. \\
& \left.+\delta_{1} h^{\gamma_{2}}(x) \times k^{1-\gamma_{1}}(x) k^{\prime}(x)\right) y^{\prime}
\end{aligned}
$$

Since $\gamma_{1}=\alpha+\rho, \gamma_{2}=\beta+\sigma, \delta_{1}=\alpha-\rho, \delta_{2}=\sigma-\beta$.

$$
\left[\mathrm{D}_{\mathrm{h}, \mathrm{k}, \mathrm{l}}^{\alpha, \beta}, \mathrm{D}_{\mathrm{h}, \mathrm{k}, \mathrm{l}}^{\alpha, \beta}\right]=0 \text {, }
$$

and

$$
\left[\mathrm{D}_{\mathrm{h}, \mathrm{k}, \mathrm{l}}^{\alpha, \beta}, \mathrm{D}_{\mathrm{h}, \mathrm{k}, \mathrm{l}}^{\rho, \sigma}\right]=-\left[\mathrm{D}_{\mathrm{h}, \mathrm{k}, \mathrm{l}}^{\rho, \sigma}, \mathrm{D}_{\mathrm{h}, \mathrm{k}, \mathrm{l}}^{\alpha, \beta}\right] .
$$

Using the result from Eq. (31):

$$
\begin{aligned}
{\left[\mathrm{D}_{\mathrm{h}, \mathrm{k}, 1}^{\mathrm{a}, \mathrm{b}},[\right.} & \left.\left.\mathrm{D}_{\mathrm{h}, \mathrm{k}, 1}^{\mathrm{c}, \mathrm{d}}, \mathrm{D}_{\mathrm{h}, \mathrm{k}, 1}^{\mathrm{m}, \mathrm{n}}\right]\right] y=\mathrm{D}_{\mathrm{h}, \mathrm{k}, \mathrm{l}}^{\mathrm{a}, \mathrm{b}}\left[\mathrm{D}_{\mathrm{h}, \mathrm{k}, \mathrm{l}}^{\mathrm{c}, \mathrm{d}}, \mathrm{D}_{\mathrm{h}, \mathrm{k}, 1}^{\mathrm{m}, \mathrm{n}}\right] y \\
& -\left[\mathrm{D}_{\mathrm{h}, \mathrm{k}, 1}^{\mathrm{c}, \mathrm{d}}, \mathrm{D}_{\mathrm{h}, \mathrm{k}, 1}^{\mathrm{m}, \mathrm{n}}\right] \mathrm{D}_{\mathrm{h}, \mathrm{k}, \mathrm{l}}^{\mathrm{a}, \mathrm{b}}[y] \\
& =k^{-a-c-m+1}(x)\left(-h^{b+d+n-2}(x)\right) \\
& \times\left(h ^ { 2 } ( x ) \left(a^{2}+a(m-2)\right.\right. \\
& -m(c+m)+c+m)\left[k^{\prime}(x)\right]^{2} y^{\prime}(x) \\
& +h(x) k(x)\left(y^{\prime}(x)((m-a) h(x)\right. \\
& \times k^{\prime \prime}(x)-h^{\prime}(x) k^{\prime}(x)(b(2 a+m-3) \\
& +n(a-c-2 m+2)+d \\
& \left.\times(-m)+d))+h(x)(-2 a+c+m) k^{\prime}(x) y^{\prime \prime}(x)\right) \\
& +k^{2}(x)\left(y ^ { \prime } ( x ) \left(\left(b^{2}+b(n-1)-n(d+n)+n\right)\right.\right. \\
& \left.\times\left[h^{\prime}\right]^{2}(x)+(b-n) h(x) h^{\prime \prime}(x)\right) \\
& \left.\left.+h(x)(2 b-d-n) h^{\prime}(x) y^{\prime \prime}(x)\right)\right) .
\end{aligned}
$$

Then, we can verify that the Jacobi identity holds:

$$
\begin{gathered}
{\left[\mathrm{D}_{\mathrm{h}, \mathrm{k}, 1}^{\mathrm{a}, \mathrm{b}}\left[\mathrm{D}_{\mathrm{h}, \mathrm{k}, \mathrm{l}}^{\mathrm{c}, \mathrm{d}}, \mathrm{D}_{\mathrm{h}, \mathrm{k}, \mathrm{l}}^{\mathrm{m}, \mathrm{n}}\right]\right]+\left[\mathrm{D}_{\mathrm{h}, \mathrm{k}, \mathrm{l}}^{\mathrm{c}, \mathrm{d}},\left[\mathrm{D}_{\mathrm{h}, \mathrm{k}, \mathrm{l}}^{\mathrm{m}, \mathrm{n}}, \mathrm{D}_{\mathrm{h}, \mathrm{k}, \mathrm{l}}^{\mathrm{a}, \mathrm{b}}\right]\right]} \\
+\left[\mathrm{D}_{\mathrm{h}, \mathrm{k}, \mathrm{l}}^{\mathrm{m}, \mathrm{n}},\left[\mathrm{D}_{\mathrm{h}, \mathrm{k}, \mathrm{l}}^{\mathrm{a}, \mathrm{b}}, \mathrm{D}_{\mathrm{h}, \mathrm{k}, \mathrm{l}, \mathrm{d}}^{\mathrm{c}, \mathrm{d}}\right]=0 .\right.
\end{gathered}
$$

If we consider the commutator $\left[D_{k, l}^{\alpha}, t\right]$ acting on a function $y(x)$ :

$$
\begin{aligned}
{\left[\mathrm{D}_{\mathrm{h}, \mathrm{k}, 1}^{\alpha, \beta}, x\right] y } & =\mathrm{D}_{\mathrm{h}, \mathrm{k}, \mathrm{l}}^{\alpha, \beta}[x y]-x \mathrm{D}_{\mathrm{h}, \mathrm{k}, \mathrm{l}}^{\alpha, \beta}[y] \\
& =x \mathrm{D}_{\mathrm{h}, \mathrm{k}, \mathrm{l}}^{\alpha, \beta}[y]+y \mathrm{D}_{\mathrm{h}, \mathrm{k}, \mathrm{l}}^{\alpha, \beta}[x]-x \mathrm{D}_{\mathrm{h}, \mathrm{k}, \mathrm{l}}^{\alpha, \beta}[y] \\
& =h^{\beta}(x) k^{1-\alpha}(x) y .
\end{aligned}
$$

Thus

$$
\left[\mathrm{D}_{\mathrm{h}, \mathrm{k}, 1}^{\alpha, \beta}, x\right]=h^{\beta}(x) k^{1-\alpha}(x) .
$$

Then, we can express the generalized conformable fractional derivative acting on $y$ as

$$
\mathrm{D}_{\mathrm{h}, \mathrm{k}, \mathrm{l}}^{\alpha, \beta}[y]=\left[\mathrm{D}_{\mathrm{h}, \mathrm{k}, \mathrm{l}}^{\alpha, \beta}, x\right] y^{\prime} .
$$

So, for any differentiable function of $x$ :

$$
\left[\mathrm{D}_{\mathrm{h}, \mathrm{k}, 1}^{\alpha, \beta}, f(x)\right]=h^{\beta}(x) k^{1-\alpha}(x) f^{\prime}(x) .
$$

Using Eq. (12) and Eq. (13), we can consider the commutator $\left[I_{k, l}^{\alpha}, D_{k, l}^{\beta}\right]$

$$
\begin{aligned}
{\left[\mathrm{I}_{\mathrm{h}, \mathrm{k}, 1}^{\alpha, \beta}, \mathrm{D}_{\mathrm{h}, \mathrm{k}, 1}^{\rho, \sigma}\right] y } & =-\delta_{1} I_{h, k, l}^{\delta_{1}, \delta_{2}}\left[h^{2 \delta}(x) k^{\prime}(x) y\right] \\
& -\delta_{2} I_{h, k, l}^{\delta_{1}, \delta_{2}}\left[h^{2 \delta_{2}-1}(x) h^{\prime}(x) k(x) y\right] .
\end{aligned}
$$

\subsection{The anti-commutator for the operator $D_{h, k, 1}^{\alpha, \beta}$}

We now consider the anti-commutator by defining the operator

$$
\begin{aligned}
\widehat{C}_{\gamma_{1}, \gamma_{2}} \equiv \frac{1}{2}\left\{\mathrm{D}_{\mathrm{h}, \mathrm{k}, \mathrm{l}}^{\alpha, \beta}, \mathrm{D}_{\mathrm{h}, \mathrm{k}, \mathrm{l}}^{\rho, \sigma}\right\} & =\frac{1}{2}\left(\mathrm{D}_{\mathrm{h}, \mathrm{k}, \mathrm{l}}^{\alpha, \beta} \mathrm{D}_{\mathrm{h}, \mathrm{k}, \mathrm{l}}^{\rho, \sigma}\right. \\
& \left.+\mathrm{D}_{\mathrm{h}, \mathrm{k}, \mathrm{l}}^{\rho, \sigma} \mathrm{D}_{\mathrm{h}, \mathrm{k}, \mathrm{l}}^{\alpha, \beta}\right) .
\end{aligned}
$$

Using Eq. (8) and operating $\widehat{C}_{\gamma}$ on a function $y(x)$ :

$$
\begin{aligned}
\widehat{C}_{\gamma_{1}, \gamma_{2}} y & =\frac{1}{2}\left(h^{\gamma_{2}}(x) k^{2-\gamma_{1}}(x) y^{\prime \prime}\right. \\
& +\left(\sigma h^{\gamma_{2}-1}(x) h^{\prime}(x) k^{2-\gamma_{1}}(x)\right. \\
& \left.\left.+(1-\rho) h^{\gamma_{2}}(x) k^{1-\gamma_{1}}(x) k^{\prime}(x)\right) y^{\prime}\right) \\
& +\frac{1}{2}\left(h^{\gamma_{2}}(x) k^{2-\gamma_{1}}(x) y^{\prime \prime}\right. \\
& +\left(\beta h^{\gamma_{2}-1}(x) h^{\prime}(x) k^{2-\gamma_{1}}(x)\right. \\
& \left.\left.+(1-\alpha) h^{\gamma_{2}}(x) k^{1-\gamma_{1}}(x) k^{\prime}(x)\right) y^{\prime}\right) \\
& =h^{\gamma_{2}}(x) k^{2-\gamma_{1}}(x) y^{\prime \prime} \\
& +\frac{1}{2}\left(\gamma_{2} h^{\gamma_{2}-1}(x) h^{\prime}(x) k^{2-\gamma_{1}}(x)\right. \\
& \left.+\left(2-\gamma_{1}\right) h^{\gamma_{2}}(x) k^{1-\gamma_{1}}(x) k^{\prime}(x)\right) y^{\prime} .
\end{aligned}
$$

We may consider the case when $\alpha=\rho$ and $\beta=\sigma$, then,

$$
\widehat{C}_{2 \alpha, 2 \beta} y=\frac{1}{2}\left\{\mathrm{D}_{\mathrm{h}, \mathrm{k}, \mathrm{l}}^{\alpha, \beta}, \mathrm{D}_{\mathrm{h}, \mathrm{k}, \mathrm{l}}^{\alpha, \beta}\right\}=\left(\mathrm{D}_{\mathrm{h}, \mathrm{k}, \mathrm{l}}^{\alpha, \beta}\right)^{2} .
$$

If $\alpha=\beta=1 / 2$ :

$$
\begin{aligned}
\widehat{C}_{1,1} y & =h(x) k(x) y^{\prime \prime} \\
& +\frac{1}{2}\left(h^{\prime}(x) k(x)-h(x) k^{\prime}(x)\right) y^{\prime} .
\end{aligned}
$$


4.6.1. Some properties for $\widehat{C}_{\gamma_{1}, \gamma_{2}} y$

Lets first consider the homogeneous equation for $\alpha=\rho$ and $\beta=\sigma$,

$$
\widehat{C}_{2 \alpha, 2 \beta} y=0 \text {. }
$$

Then Eq. (42) becomes:

$$
\begin{gathered}
h^{2 \beta}(x) k^{2-2 \alpha}(x) y^{\prime \prime}+\left(\beta h^{2 \beta-1}(x) h^{\prime}(x) k^{2-2 \alpha}(x)\right. \\
\left.+(1-\alpha) h^{2 \beta}(x) k^{1-2 \alpha}(x) k^{\prime}(x)\right) y^{\prime}=0,
\end{gathered}
$$

which has solution:

$$
y(x)=C_{2}+\int^{x} C_{1} h^{-\beta}(t) k^{\alpha-1}(t) \mathrm{d} t .
$$

Lets now consider the constant equation

$$
\widehat{C_{2 \alpha, 2 \beta}} y=\Lambda
$$

Equation (42) becomes

$$
\begin{aligned}
& x^{2} k^{-2 \alpha}(x) y^{\prime \prime}+\left(t k^{-2 \alpha}(x)\right. \\
& \left.-\alpha x^{2} k^{-(2 \alpha+1)}(x)\right) y^{\prime}=\Lambda,
\end{aligned}
$$

which has the solution:

$$
\begin{aligned}
y(x) & =c_{2}+\int\left(c_{1} e^{\int \frac{\alpha s^{2}-k(s) s}{k(s) s^{2}} \mathrm{~d} s}+e^{\int x \frac{\alpha s^{2}-k(s) s}{k(s) s^{2}} \mathrm{~d} s}\right. \\
& \left.\times \int \frac{e^{\int \frac{\alpha s^{2}-k(s) s}{k(s) s^{2}} \mathrm{~d} s} \Lambda k^{2 \alpha}(z)}{z^{2}} \mathrm{~d} z\right) \mathrm{d} t,
\end{aligned}
$$

where $c_{1}$ and $c_{2}$ are constants to be determined by boundary conditions.

\subsubsection{Self-adjoint operator of $\widehat{C}_{2 \alpha, 2 \beta}$}

The operator $\widehat{C}_{2 \alpha, 2 \beta}$ is not self-adjoint, but it can be made so by multiplying by an integrating factor $W(x)$,

$$
\begin{aligned}
W(x) & =\frac{1}{h^{2 \beta}(x) k^{2-2 \alpha}(x)} \\
& \times e^{\int^{x} \frac{\beta h^{2 \beta-1}(x) h^{\prime}(x) k^{2-2 \alpha}(x)+(1-\alpha) h^{2 \beta}(x) k^{1-2 \alpha}(x) k^{\prime}(x)}{h^{2 \beta}(x) k^{2-2 \alpha}(x)} d x} \\
& =\frac{1}{h^{2 \beta}(x) k^{2-2 \alpha}(x)} e^{\int^{x} \beta \frac{h^{\prime}(x)}{h(x)}+(1-\alpha) \frac{k^{\prime}(x)}{k(x)} d x} \\
& =h^{-\beta}(x) k^{\alpha-1}(x) .
\end{aligned}
$$

We define:

$$
\begin{aligned}
\widehat{A}_{2 \alpha, 2 \beta} & \equiv W(x) \widehat{C}_{2 \alpha, 2 \beta}=\left(h^{-\beta}(x) k^{\alpha-1}(x)\right)\left(h^{2 \beta}(x) k^{2-2 \alpha}(x)\right. \\
& \times \frac{d^{2}}{d x^{2}}+\left[\beta h^{2 \beta-1}(x) h^{\prime}(x) k^{2-2 \alpha}(x)\right. \\
& \left.\left.+(1-\alpha) h^{2 \beta}(x) k^{1-2 \alpha}(x) k^{\prime}(x)\right] \frac{d}{d x}\right) \\
& =h^{\beta}(x) k^{1-\alpha}(x) \frac{d^{2}}{d x^{2}}+\left(\beta h^{\beta-1}(x) h^{\prime}(x)\right. \\
& \times k^{1-\alpha}(x)+(1-\alpha) h^{\beta}(x) k^{-\alpha}(x) \\
& \left.\times k^{\prime}(x)\right) \frac{d}{d x}=\frac{d}{d x}\left(h^{\beta}(x) k^{1-\alpha}(x) \frac{d}{d x}\right)
\end{aligned}
$$

\subsubsection{Differential equations for $\widehat{A}_{2 \alpha, 2 \beta}$}

Now that we have defined $\widehat{A}_{2 \alpha, 2 \beta}$, we solve two differential equations using this operator.

1. Homogeneous equation

$$
\widehat{A}_{2 \alpha, 2 \beta} y=0,
$$

which solution is:

$$
y(x)=c_{1} \int^{x} h^{-\beta}(s) k^{\alpha-1}(s) \mathrm{d} s+c_{2},
$$

where $c_{1}$ and $c_{2}$ are constants to be determined by boundary conditions.

2. Equation including a constant inhomogeneous term

$$
\begin{aligned}
\widehat{A}_{2 \alpha, 2 \beta} y & =\kappa, \\
y(x) & =\int_{1}^{x}\left(c_{1}+\kappa s\right) h^{-\beta}(s) k^{\alpha-1}(s) \mathrm{d} s+c_{2},
\end{aligned}
$$

where $c_{1}$ and $c_{2}$ are constants to be determined by boundary conditions, and $\kappa$ is a constant term.

Example:

Setting $h(x)=x^{m}$ and $k(x)=x^{n}$, yields the solution:

$$
\begin{aligned}
y(x) & =\frac{c_{1} x^{1+n(\alpha-1)-m \beta}}{1+n(\alpha-1)-m \beta} \\
& +\frac{\kappa x^{2+n(\alpha-1)-m \beta}}{2+n(\alpha-1)-m \beta}+c_{2},
\end{aligned}
$$

where $n, m \in \mathbb{Z}_{>0}$.

We can re-express this solution in the from:

$$
\begin{aligned}
y_{G, q_{1}, q_{2}, q_{3}}(x) & =q_{1}(\alpha, \beta, m, n) x^{G(\alpha, \beta, m, n)} \\
& +q_{2}(\alpha, \beta, m, n) x^{1+G(\alpha, \beta, m, n)}+q_{3},
\end{aligned}
$$



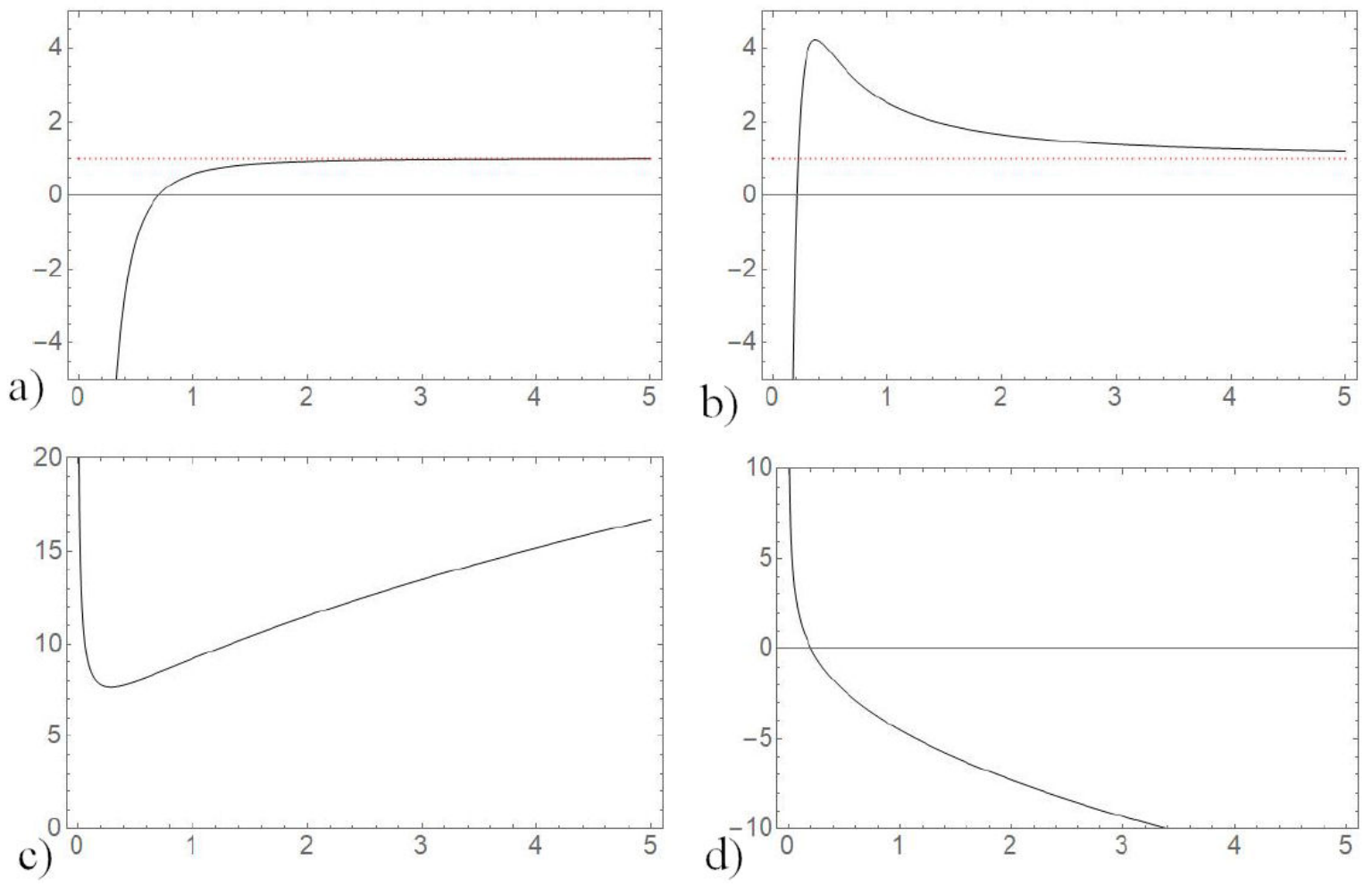

FIGURE 1. Plots of different values of $\alpha, \beta, m, n, c_{1}, c_{2}$, and $\kappa$ for Eq. (58). [22].

where:

$$
\begin{aligned}
G(\alpha, \beta, m, n) & =1+n(\alpha-1)-m \beta \\
q_{1}(\alpha, \beta, m, n) & =\frac{c_{1}}{G_{1}(\alpha, \beta, m, n)} \\
q_{2}(\alpha, \beta, m, n) & =\frac{\kappa}{1+G(\alpha, \beta, m, n)} \\
q_{3}(\alpha, \beta, m, n) & =c_{2} .
\end{aligned}
$$

By the law of trichotomy, Eq. (58) leaves us only with three non-singular solutions of the form:

(1) $G(\alpha, \beta, m, n)<1+G(\alpha, \beta, m, n)<0$,

(2) $G_{1}(\alpha, \beta, m, n)<0<1+G(\alpha, \beta, m, n)$,

(3) $0<G(\alpha, \beta, m, n)<1+G(\alpha, \beta, m, n)$.

The next table shows some cases of these solutions of Eq. (58):

(1) Figure 1a), obtained by setting $\alpha=0.5, \beta=0.8$, $m=3, n=2, c_{1}=1, c_{2}=1, \kappa=0$, has an asymptotic behaviour determined by the value of $c_{2}$, which is indicated by the dotted line.

(2) Figure 1b), obtained by setting $\alpha=0.5, \beta=0.8$, $m=3, n=2, c_{1}=1, c_{2}=1, \kappa=-2.7$, has an asymptotic behaviour determined by the value of $c_{2}$, which is indicated by the dotted line and the maximum value of the function is determined by the value $c_{1}$.
(3) Figure 1c), obtained by setting $\alpha=0.7, \beta=0.3$, $m=3, n=2, c_{1}=-1, c_{2}=0.2, \kappa=3.5$. In this case, the function diverges to $+\infty$ both at $x=0$ and at $x \rightarrow \infty$.

(4) Figure 1d), obtained by setting $\alpha=0.7, \beta=0.3$, $m=3, n=2, c_{1}=-0.5, c_{2}=0.5, \kappa=-3$. The function now diverges to $+\infty$ at $x=0$ and to $-\infty$ at $x \rightarrow-\infty$.

For $G>0$ is an interesting family of elemental functions since:

- The value $x=0$ is a local minimum or maximum in the domain $\mathbb{R}^{+} \bigcup\{0\}$.

- By extending the domain to negative numbers, the function takes complex values, it becomes multivalued and the concept of maximum, and minimum no longer makes sense.

- At least the first two derivatives are null in the minimum $x=0$

- When $G=N$ is a natural positive exponent, the $y \in C^{\infty}$ in $\mathbb{R}^{+} \bigcup\{0\}$. When $G$ is not a natural number, there is a first non-null derivative a $x=0$, which is infinite. 


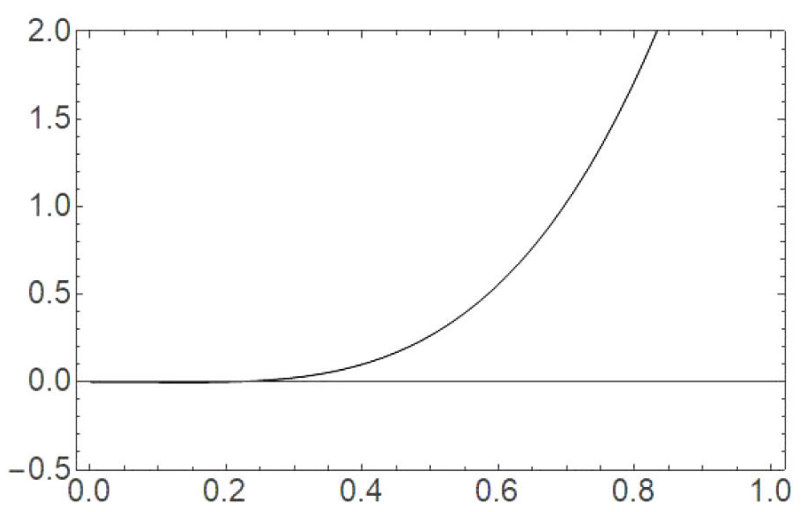

Figure 2. Plot of Eq. (64). [22].

By setting $c_{1}=-2.5, c_{2}=0, \kappa=17.5, n=m=1$, $\alpha=0.3, \beta=0.5$ in Eq. (57), we get:

$$
\begin{aligned}
& y(x)=q_{1} x^{G_{1}}+q_{2} x^{1+G_{1}}+q_{3}, \\
& y(x)=-x^{2.5}+5 x^{3.5}
\end{aligned}
$$

which is an interesting example of an elementary function (see Fig. 2).

\section{Results of the $D_{h, k, l}^{\alpha, \beta}$ derivative for the Sturm-Liouville eigenvalue problem}

We consider the fractional extension of the Sturm-Liouville eigenvalue problem.

$$
\begin{gathered}
\mathrm{D}_{\mathrm{h}, \mathrm{k}, \mathrm{l}}^{\alpha, \beta}\left(p(x) \mathrm{D}_{\mathrm{h}, \mathrm{k}, \mathrm{l}}^{\alpha, \beta} y(x)\right)+q(x) y(x)=-\lambda w(x) y(x) \\
0<\alpha \leq 1,0<\beta \leq 1, \quad a<x<b
\end{gathered}
$$

where $p, \mathrm{D}_{\mathrm{h}, \mathrm{k}, \mathrm{l}}^{\alpha, \beta} p, q$ and the weight functions $w$ are continuous on $(a, b), p(x)>0$, and $w(x)>0$, on $[a, b]$, and the fractional derivative $\mathrm{D}_{\mathrm{h}, \mathrm{k}, \mathrm{l}}^{\alpha, \beta}$ is the generalized conformable fractional derivative. We consider Eq. (65) with boundary conditions

$$
\begin{aligned}
& c_{1} y(a)+c_{2} y^{\prime}(a)=0, \quad c_{1}^{2}+c_{2}^{2}>0, \\
& r_{1} y(b)+r_{2} y^{\prime}(b)=0, \quad r_{1}^{2}+r_{2}^{2}>0 .
\end{aligned}
$$

If $\mathrm{D}_{\mathrm{h}, \mathrm{k}, \mathrm{l}}^{\alpha, \beta} \mathrm{D}_{\mathrm{h}, \mathrm{k}, \mathrm{l}}^{\alpha, \beta} y$ is continuous on $[a, b]$, then we say that $\mathrm{y}$ is $2 \gamma$-continuously differentiable on $[a, b]$. Let

$$
\mathrm{L}_{\mathrm{h}, \mathrm{k}, \mathrm{l}}^{\alpha, \beta}(\mathrm{y})=\mathrm{D}_{\mathrm{h}, \mathrm{k}, \mathrm{l}}^{\alpha, \beta}\left(p(x) \mathrm{D}_{\mathrm{h}, \mathrm{k}, \mathrm{l}}^{\alpha, \beta} y(x)\right)+q(x) y(x) .
$$

Then we may write the fractional Sturm-Liouville eigenvalue problem as

$$
\mathrm{L}_{\mathrm{h}, \mathrm{k}, \mathrm{l}}^{\alpha, \beta}(\mathrm{y})=-\lambda w(x) y(x) .
$$

\section{Theorem 5.1 (New Lagrange identity).}

Letting $y_{1}, y_{2}$ be $2 \gamma$-continuously differentiable on $[a, b]$, then the following holds:

$$
\begin{aligned}
& \int_{a}^{b}\left(y_{2} \mathrm{~L}_{\mathrm{h}, \mathrm{k}, \mathrm{l}}^{\alpha, \beta}\left(\mathrm{y}_{1}\right)-y_{1} \mathrm{~L}_{\mathrm{h}, \mathrm{k}, \mathrm{l}}^{\alpha, \beta}\left(\mathrm{y}_{2}\right)\right) \mathrm{d} \gamma(x) \\
& \quad=\left.\left.\left[p(x)\left(y_{2} \mathrm{D}_{\mathrm{h}, \mathrm{k}, \mathrm{l}}^{\alpha, \beta} y_{1}-y_{1} \mathrm{D}_{\mathrm{h}, \mathrm{k}, \mathrm{l}}^{\alpha, \beta} y_{2}\right)\right]\right|_{a} ^{b} f(x)\right|_{a} ^{b}
\end{aligned}
$$

proof

Let $F_{4}(x, \alpha, \beta)=y_{2} \mathrm{~L}_{\mathrm{h}, \mathrm{k}, \mathrm{l}}^{\alpha, \beta}\left(\mathrm{y}_{1}\right)-y_{1} \mathrm{~L}_{\mathrm{h}, \mathrm{k}, \mathrm{l}}^{\alpha, \beta}\left(\mathrm{y}_{2}\right)$, then:

$$
\begin{aligned}
& \int_{a}^{b} F_{4}(x, \alpha, \beta) \mathrm{d} \gamma(x) \\
& \quad=\int_{a}^{b} y_{2} \mathrm{D}_{\mathrm{h}, \mathrm{k}, \mathrm{l}}^{\alpha, \beta}\left(p(x) \mathrm{D}_{\mathrm{h}, \mathrm{k}, \mathrm{l}}^{\alpha, \beta} y_{1}\right) \mathrm{d} \gamma(x) \\
& \quad-\int_{a}^{b} y_{1} \mathrm{D}_{\mathrm{h}, \mathrm{k}, 1}^{\alpha, \beta}\left(p(x) \mathrm{D}_{\mathrm{h}, \mathrm{k}, \mathrm{l}}^{\alpha, \beta} y_{2}\right) \mathrm{d} \gamma(x) \\
& =\left.p(x) y_{2} \mathrm{D}_{\mathrm{h}, \mathrm{k}, 1}^{\alpha, \beta} y_{1}\right|_{a} ^{b}-\int_{a}^{b} p(x) \mathrm{D}_{\mathrm{h}, \mathrm{k}, 1}^{\alpha, \beta} y_{1} \mathrm{D}_{\mathrm{h}, \mathrm{k}, \mathrm{l}}^{\alpha, \beta} y_{2} \mathrm{~d} \gamma(x) \\
& -\left.p(x) y_{1} \mathrm{D}_{\mathrm{h}, \mathrm{k}, 1}^{\alpha, \beta} y_{2}\right|_{a} ^{b}+\int_{a}^{b} p(x) \mathrm{D}_{\mathrm{h}, \mathrm{k}, 1}^{\alpha, \beta} y_{1} \mathrm{D}_{\mathrm{h}, \mathrm{k}, 1}^{\alpha, \beta} y_{2} \mathrm{~d} \gamma(x) \\
& =\left.\left[p(x)\left(y_{2} \mathrm{D}_{\mathrm{h}, \mathrm{k}, 1}^{\alpha, \beta} y_{1}-y_{1} \mathrm{D}_{\mathrm{h}, \mathrm{k}, \mathrm{l}}^{\alpha, \beta} y_{2}\right)\right]\right|_{a} ^{b} \cdot
\end{aligned}
$$

\section{Lemma 5.2}

Let $y_{1}$ and $y_{2}$ in $C^{1}[a, b]$, which satisfy the boundary conditions (66) and (67). Then it holds that:

$$
\left.\left[p(x)\left(y_{2} \mathrm{D}_{\mathrm{h}, \mathrm{k}, \mathrm{l}}^{\alpha, \beta} y_{1}-y_{1} \mathrm{D}_{\mathrm{h}, \mathrm{k}, \mathrm{l}}^{\alpha, \beta} y_{2}\right)\right]\right|_{a} ^{b}=0 .
$$

Proof. then:

$$
\text { Let } F_{5}(x, \alpha, \beta)=p(x)\left(y_{2} \mathrm{D}_{\mathrm{h}, \mathrm{k}, \mathrm{l}}^{\alpha, \beta} y_{1}-y_{1} \mathrm{D}_{\mathrm{h}, \mathrm{k}, \mathrm{l}}^{\alpha, \beta} y_{2}\right) \text {, }
$$

$$
\begin{aligned}
\left.F_{5}(x, \alpha, \beta)\right|_{a} ^{b} & =p(b)\left(y_{2}(b) \mathrm{D}_{\mathrm{h}, \mathrm{k}, \mathrm{l}}^{\alpha, \beta} y_{1}(b)\right. \\
& \left.-y_{1}(b) \mathrm{D}_{\mathrm{h}, \mathrm{k}, 1}^{\alpha, \beta} y_{2}(b)\right) \\
& -p(a)\left(y_{2}(a) \mathrm{D}_{\mathrm{h}, \mathrm{k}, 1}^{\alpha, \beta} y_{1}(a)\right. \\
& \left.-y_{1}(a) \mathrm{D}_{\mathrm{h}, \mathrm{k}, \mathrm{l}}^{\alpha, \beta} y_{2}(a)\right) .
\end{aligned}
$$

Since $c_{1}^{2}+c_{2}^{2}>0$ and $r_{1}^{2}+r_{2}^{2}>0$, we first assume that $c_{1} \neq 0$ and $r_{1} \neq 0$, then:

$$
\begin{aligned}
& y(a)=-\frac{c_{2}}{c_{1}} y^{\prime}(a), \\
& y(b)=-\frac{r_{2}}{r_{1}} y^{\prime}(b) .
\end{aligned}
$$


Thus

$$
\begin{aligned}
y_{2}(b) & \mathrm{D}_{\mathrm{h}, \mathrm{k}, 1}^{\alpha, \beta} y_{1}(b)-y_{1}(b) \mathrm{D}_{\mathrm{h}, \mathrm{k}, \mathrm{l}}^{\alpha, \beta} y_{2}(b)=-\frac{r_{2}}{r_{1}} \\
& \times\left(y_{2}^{\prime}(b) h^{\beta}(b) k^{1-\alpha}(b) y_{1}^{\prime}(b)\right. \\
& \left.-y_{1}^{\prime}(b) h^{\beta}(b) k^{1-\alpha}(b) y_{2}^{\prime}(b)\right)=0, \\
y_{2}(a) & \mathrm{D}_{\mathrm{h}, \mathrm{k}, 1}^{\alpha, \beta} y_{1}(a)-y_{1}(a) \mathrm{D}_{\mathrm{h}, \mathrm{k}, \mathrm{l}}^{\alpha, \beta} y_{2}(a)=-\frac{c_{2}}{c_{1}} \\
& \times\left(y_{2}^{\prime}(a) h^{\beta}(a) k^{1-\alpha}(a) y_{1}^{\prime}(a)\right. \\
& \left.-y_{1}^{\prime}(a) h^{\beta}(a) k^{1-\alpha}(a) y_{2}^{\prime}(a)\right)=0 .
\end{aligned}
$$

Definition 1. We say that $f$ and $g$ are $\gamma$-orthogonal with respect to the weight function $\mu(x) \geq 0$, if

$$
\int_{a}^{b} f(x) \mu(x) g(x) \mathrm{d} \gamma(x)=0 .
$$

\section{Theorem 5.3}

The eigenfunctions of the fractional eigenvalue problem (65.66.67) corresponding to distinct eigenvalues are $\gamma$-orthogonal with respect to a weight function $w(x)$.

\section{Proof.}

Let $\lambda_{1}$ and $\lambda_{2}$ be two distinct eigenvalues, and $y_{1}$ and $y_{2}$ are the corresponding eigenfunctions, then

$$
\begin{aligned}
\mathrm{L}_{\mathrm{h}, \mathrm{k}, \mathrm{l}}^{\alpha, \beta}\left(\mathrm{y}_{1}\right) & =-\lambda_{1} w(x) y_{1}, \\
\mathrm{~L}_{\mathrm{h}, \mathrm{k}, \mathrm{l}}^{\alpha, \beta}\left(\mathrm{y}_{2}\right) & =-\lambda_{2} w(x) y_{2}, \\
y_{2} \mathrm{~L}_{\mathrm{h}, \mathrm{k}, \mathrm{l}}^{\alpha, \beta}\left(\mathrm{y}_{1}\right)-y_{1} \mathrm{~L}_{\mathrm{h}, \mathrm{k}, \mathrm{l}}^{\alpha, \beta}\left(\mathrm{y}_{2}\right) & =-\left(\lambda_{1}-\lambda_{2}\right) w(x) y_{1} y_{2}, \\
-\left(\lambda_{1}-\lambda_{2}\right) & \int_{a}^{b} w(x) y_{1} y_{2} \mathrm{~d} \gamma(x) \\
& =\int_{a}^{b} y_{2} \mathrm{~L}_{\mathrm{h}, \mathrm{k}, \mathrm{l}}^{\alpha, \beta}\left(\mathrm{y}_{1}\right) \mathrm{d} \gamma(x) \\
& -\int_{a}^{b} y_{1} \mathrm{~L}_{\mathrm{h}, \mathrm{k}, \mathrm{l}}^{\alpha, \beta}\left(\mathrm{y}_{2}\right) \mathrm{d} \gamma(x) .
\end{aligned}
$$

From the New Lagrange identity Eq. (70) and Lemma 5.2, Eq. (71):

$$
\left(\lambda_{1}-\lambda_{2}\right) \int_{a}^{b} w(x) y_{1} y_{2} \mathrm{~d} \gamma(x)=0 .
$$

Since $\lambda_{1} \neq \lambda_{2}$

$$
\int_{a}^{b} w(x) y_{1} y_{2} \mathrm{~d} \gamma(x)=0 .
$$

\section{Theorem 5.4}

The eigenvalues of the fractional eigenvalue problem (65.66.67) are real.

Proof. Let $y$ be a solution of the fractional SturmLiouville eigenvalue problem. Taking the complex conjugate of Eq. (68) and Eq. (69) and using the fact that $p(x), q(x)$, and $w(x)$ are real-valued functions, we have:

$$
\begin{aligned}
\mathrm{L}_{\mathrm{h}, \mathrm{k}, \mathrm{l}}^{\alpha, \beta}(\overline{\mathrm{y}}) & =\mathrm{D}_{\mathrm{h}, \mathrm{k}, \mathrm{l}}^{\alpha, \beta}\left(p(x) \overline{\mathrm{D}_{\mathrm{h}, \mathrm{k}, \mathrm{l}}^{\alpha, \beta} y}\right)+q(x) \bar{y} \\
& =-\lambda w(x) \bar{y} \\
c_{1} \bar{y}(a) & +c_{2} \overline{y^{\prime}}(a)=0 \\
r_{1} \bar{y}(a) & +r_{2} \overline{y^{\prime}}(b)=0 \\
\bar{y} \mathrm{~L}_{\mathrm{h}, \mathrm{k}, \mathrm{l}}^{\alpha, \beta}(\mathrm{y})-y \mathrm{~L}_{\mathrm{h}, \mathrm{k}, \mathrm{l}}^{\alpha, \beta}(\overline{\mathrm{y}}) & =-(\lambda-\bar{\lambda}) w(x) y \bar{y} \\
& -(\lambda-\bar{\lambda}) w(x)\|y(x)\|^{2}
\end{aligned}
$$

Then, from Lemma 5.2, Eq. (71) and Theorem 5.3,

$$
(\lambda-\bar{\lambda}) \int_{a}^{b} w(x)\|y(x)\|^{2} \mathrm{~d} \gamma(x)=0 .
$$

Thus

$$
\lambda=\bar{\lambda}
$$

\section{Definition 2.}

Let $f$ and $g$ be $\gamma$-differentiable. The new Wronskian function is defined by

$$
\mathrm{W}_{\mathrm{h}, \mathrm{k}, \mathrm{l}}^{\alpha, \beta}(\mathrm{f}, \mathrm{g})=f \mathrm{D}_{\mathrm{h}, \mathrm{k}, \mathrm{l}}^{\alpha, \beta} g-g \mathrm{D}_{\mathrm{h}, \mathrm{k}, \mathrm{l}}^{\alpha, \beta} f .
$$

\section{Theorem 5.5}

Let $y_{1}$ and $y_{2}$ be $2 \gamma$-continuously differentiable on [a,b]; they are linearly independent solutions of (65), then:

$$
\mathrm{W}_{\mathrm{h}, \mathrm{k}, \mathrm{l}}^{\alpha, \beta}\left(\mathrm{y}_{1}, \mathrm{y}_{2}\right)=\frac{\mathrm{W}_{\mathrm{h}, \mathrm{k}, \mathrm{l}}^{\alpha, \beta}\left(\mathrm{y}_{1}, \mathrm{y}_{2}\right)(a) p(a)}{p(x)} .
$$

\section{Proof.}

$$
\begin{aligned}
\mathrm{D}_{\mathrm{h}, \mathrm{k}, \mathrm{l}}^{\alpha, \beta} & \mathrm{W}_{\mathrm{h}, \mathrm{k}, \mathrm{l}}^{\alpha, \beta}\left(\mathrm{y}_{1}, \mathrm{y}_{2}\right)=\mathrm{D}_{\mathrm{h}, \mathrm{k}, \mathrm{l}}^{\alpha, \beta}\left[y_{1} \mathrm{D}_{\mathrm{h}, \mathrm{k}, \mathrm{l}}^{\alpha, \beta} y_{2}-y_{2} \mathrm{D}_{\mathrm{h}, \mathrm{k}, \mathrm{l}}^{\alpha, \beta} y_{1}\right] \\
& =y_{1} \mathrm{D}_{\mathrm{h}, \mathrm{k}, \mathrm{l}}^{\alpha, \beta} \mathrm{D}_{\mathrm{h}, \mathrm{k}, \mathrm{l}}^{\alpha, \beta} y_{2}+\mathrm{D}_{\mathrm{h}, \mathrm{k}, \mathrm{l}}^{\alpha, \beta} y_{1} \mathrm{D}_{\mathrm{h}, \mathrm{k}, \mathrm{l}}^{\alpha, \beta} y_{2} \\
& -y_{2} \mathrm{D}_{\mathrm{h}, \mathrm{k}, \mathrm{l}}^{\alpha, \beta} \mathrm{D}_{\mathrm{h}, \mathrm{k}, \mathrm{l}}^{\alpha, \beta} y_{1}-\mathrm{D}_{\mathrm{h}, \mathrm{k}, \mathrm{l}}^{\alpha, \beta} y_{1} \mathrm{D}_{\mathrm{h}, \mathrm{k}, \mathrm{l}}^{\alpha, \beta} y_{2} \\
& =y_{1} \mathrm{D}_{\mathrm{h}, \mathrm{k}, \mathrm{l}}^{\alpha, \beta} \mathrm{D}_{\mathrm{h}, \mathrm{k}, \mathrm{l}}^{\alpha, \beta} y_{2}-y_{2} \mathrm{D}_{\mathrm{h}, \mathrm{k}, \mathrm{l}}^{\alpha, \beta} \mathrm{D}_{\mathrm{h}, \mathrm{k}, \mathrm{l}}^{\alpha, \beta} y_{1}
\end{aligned}
$$

Analogously, applying the product rule to Eq. (65)

$$
\begin{aligned}
\mathrm{D}_{\mathrm{h}, \mathrm{k}, \mathrm{l}}^{\alpha, \beta} \mathrm{D}_{\mathrm{h}, \mathrm{k}, \mathrm{l}}^{\alpha, \beta} y & =-\frac{1}{p}\left(\mathrm{D}_{\mathrm{h}, \mathrm{k}, \mathrm{l}}^{\alpha, \beta} p \mathrm{D}_{\mathrm{h}, \mathrm{k}, \mathrm{l}}^{\alpha, \beta} y\right. \\
& +(q+\lambda w) y) .
\end{aligned}
$$


Substituting into Eq. (92):

$$
\begin{aligned}
\mathrm{D}_{\mathrm{h}, \mathrm{k}, \mathrm{l}}^{\alpha, \beta} & \mathrm{W}_{\mathrm{h}, \mathrm{k}, \mathrm{l}}^{\alpha, \beta}\left(\mathrm{y}_{1}, \mathrm{y}_{2}\right) \\
& =-\frac{y_{1}}{p}\left(\mathrm{D}_{\mathrm{h}, \mathrm{k}, \mathrm{l}}^{\alpha, \beta} p \mathrm{D}_{\mathrm{h}, \mathrm{k}, \mathrm{l}}^{\alpha, \beta} y_{2}+(q+\lambda w) y_{2}\right) \\
& +\frac{y_{2}}{p}\left(\mathrm{D}_{\mathrm{h}, \mathrm{k}, \mathrm{l}}^{\alpha, \beta} p \mathrm{D}_{\mathrm{h}, \mathrm{k}, \mathrm{l}}^{\alpha, \beta} y_{1}+(q+\lambda w) y_{1}\right) \\
& =-\frac{\mathrm{D}_{\mathrm{h}, \mathrm{k}, \mathrm{l}}^{\alpha, \beta} p}{p}\left(y_{1} \mathrm{D}_{\mathrm{h}, \mathrm{k}, \mathrm{l}}^{\alpha, \beta} y_{2}-y_{2} \mathrm{D}_{\mathrm{h}, \mathrm{k}, \mathrm{l}}^{\alpha, \beta} y_{1}\right) \\
& =-\frac{\mathrm{D}_{\mathrm{h}, \mathrm{k}, \mathrm{l}}^{\alpha, \beta}}{p} \mathrm{~W}_{\mathrm{h}, \mathrm{k}, \mathrm{l}}^{\alpha, \beta}\left(\mathrm{y}_{1}, \mathrm{y}_{2}\right) .
\end{aligned}
$$

Solving the differential equation,

$$
\mathrm{W}_{\mathrm{h}, \mathrm{k}, \mathrm{l}}^{\alpha, \beta}\left(\mathrm{y}_{1}, \mathrm{y}_{2}\right)=\frac{c}{p} .
$$

Then:

$$
\mathrm{W}_{\mathrm{h}, \mathrm{k}, \mathrm{l}}^{\alpha, \beta}\left(\mathrm{y}_{1}, \mathrm{y}_{2}\right)(a)=\frac{c}{p(a)} .
$$

Thus:

$$
\mathrm{W}_{\mathrm{h}, \mathrm{k}, 1}^{\alpha, \beta}\left(\mathrm{y}_{1}, \mathrm{y}_{2}\right)=\frac{\mathrm{W}_{\mathrm{h}, \mathrm{k}, 1}^{\alpha, \beta}\left(\mathrm{y}_{1}, \mathrm{y}_{2}\right)(a) p(a)}{p(x)} .
$$

\section{Theorem 5.5}

The eigenvalues of the fractional eigenvalue problem (65. 66. 67) are simple.

Proof. Let $y_{1}$ and $y_{2}$ be two eigenfunctions for the same eigenvalue $\lambda$ :

$$
\begin{aligned}
y_{2} \mathrm{~L}_{\mathrm{h}, \mathbf{k}, 1}^{\alpha, \beta}\left(\mathrm{y}_{1}\right)-y_{1} \mathrm{~L}_{\mathrm{h}, \mathbf{k}, 1}^{\alpha, \beta}\left(\mathrm{y}_{2}\right) & =-(\lambda-\lambda) w(x) y_{1} y_{2}, \\
y_{2} L\left(y_{1}, \alpha\right)-y_{1} L\left(y_{2}, \alpha\right) & =0 .
\end{aligned}
$$

From Eq. (70)

$$
\begin{aligned}
y_{2} \mathrm{D}_{\mathrm{h}, \mathrm{k}, \mathrm{l}}^{\alpha, \beta}\left(p(x) \mathrm{D}_{\mathrm{h}, \mathrm{k}, \mathrm{l}}^{\alpha, \beta} y_{1}\right) \\
\quad-y_{1} \mathrm{D}_{\mathrm{h}, \mathrm{k}, \mathrm{l}}^{\alpha, \beta}\left(p(x) \mathrm{D}_{\mathrm{h}, \mathrm{k}, \mathrm{l}}^{\alpha, \beta} y_{2}\right)=0 .
\end{aligned}
$$

Using the fractional product rule and factoring terms:

$$
\begin{aligned}
p(x)\left(y_{2} \mathrm{D}_{\mathrm{h}, \mathrm{k}, \mathrm{l}}^{\alpha, \beta} \mathrm{D}_{\mathrm{h}, \mathrm{k}, \mathrm{l}}^{\alpha, \beta} y_{1}-y_{1} \mathrm{D}_{\mathrm{h}, \mathrm{k}, \mathrm{l}}^{\alpha, \beta} \mathrm{D}_{\mathrm{h}, \mathrm{k}, \mathrm{l}}^{\alpha, \beta} y_{2}\right) & \\
+\mathrm{D}_{\mathrm{h}, \mathrm{k}, \mathrm{l}}^{\alpha, \beta} p(x)\left(y_{2} \mathrm{D}_{\mathrm{h}, \mathrm{k}, \mathrm{l}}^{\alpha, \beta} y_{1}-y_{1} \mathrm{D}_{\mathrm{h}, \mathrm{k}, 1}^{\alpha, \beta} y_{2}\right) & =0 \\
\mathrm{D}_{\mathrm{h}, \mathrm{k}, \mathrm{l}}^{\alpha, \beta}\left(p(x)\left[y_{2} \mathrm{D}_{\mathrm{h}, \mathrm{k}, \mathrm{l}}^{\alpha, \beta} y_{1}-y_{1} \mathrm{D}_{\mathrm{h}, \mathrm{k}, \mathrm{l}}^{\alpha, \beta} y_{2}\right]\right) & = \\
p(x)\left[y_{2} \mathrm{D}_{\mathrm{h}, \mathrm{k}, \mathrm{l}}^{\alpha, \beta} y_{1}-y_{1} \mathrm{D}_{\mathrm{h}, \mathrm{k}, \mathrm{l}}^{\alpha, \beta} y_{2}\right] & =c .
\end{aligned}
$$

Since $y_{1}$ and $y_{2}$ satisfy the same boundary conditions, $c=0$

$$
y_{2} \mathrm{D}_{\mathrm{h}, \mathrm{k}, \mathrm{l}}^{\alpha, \beta} y_{1}-y_{1} \mathrm{D}_{\mathrm{h}, \mathrm{k}, \mathrm{l}}^{\alpha, \beta} y_{2}=0 .
$$

Since $\mathrm{W}_{\mathrm{h}, \mathrm{k}, 1}^{\alpha, \beta}\left(\mathrm{y}_{1}, \mathrm{y}_{2}\right)=0$ and $y_{1}$ and $y_{2}$ are both solutions to the fractional eigenvalue problem $(65,66,67)$, then they are linearly dependent.

\section{Theorem 5.7}

(New Rayleigh Quotient) The eigenvalues $\lambda$ of the problem (65) satisfy

$$
\lambda=\frac{\int_{a}^{b} p(x)\left(\mathrm{D}_{\mathrm{h}, \mathrm{k}, \mathrm{l}}^{\alpha, \beta} y\right)^{2} \mathrm{~d} \gamma(x)-\int_{a}^{b} q(x) y^{2} \mathrm{~d} \gamma(x)-\left.p(x) y \mathrm{D}_{\mathrm{h}, \mathrm{k}, \mathrm{l}}^{\alpha, \beta} y\right|_{a} ^{b}}{\int_{a}^{b} w(x) y^{2} \mathrm{~d} \gamma(x)} .
$$

Proof. Multiplying Eq. (65) by $y$ and integrating

$$
\begin{aligned}
& \int_{a}^{b} y \mathrm{D}_{\mathrm{h}, \mathrm{k}, 1}^{\alpha, \beta}\left(p(x) \mathrm{D}_{\mathrm{h}, \mathrm{k}, 1}^{\alpha, \beta} y\right) \mathrm{d} \gamma(x) \\
& +\int_{a}^{b} q(x) y^{2} \mathrm{~d} \gamma(x)=-\lambda \int_{a}^{b} w(x) y^{2} \mathrm{~d} \gamma(x) \\
& \left.p(x) y \mathrm{D}_{\mathrm{h}, \mathrm{k}, \mathrm{l}}^{\alpha, \beta} y\right|_{a} ^{b}-\int_{a}^{b} p(x)\left(\mathrm{D}_{\mathrm{h}, \mathrm{k}, \mathrm{l}}^{\alpha, \beta} y\right)^{2} \mathrm{~d} \gamma(x) \\
& +\int_{a}^{b} q(x) y^{2} \mathrm{~d} \gamma(x)=-\lambda \int_{a}^{b} w(x) y^{2} \mathrm{~d} \gamma(x) .
\end{aligned}
$$

\subsection{The Euler-Lagrange equation}

Theorem 5.8 (Euler-Lagrange equation) Let $\mathfrak{J}$ be a function of the form

$$
\mathfrak{J}(y)=\int_{a}^{b} L\left(x, y(x), \mathrm{D}_{\mathrm{h}, \mathbf{k}, \mathrm{l}}^{\alpha, \beta} y(x)\right) \mathrm{d} \gamma(x),
$$

with $\mathrm{L} \in C^{1}\left([a, b] \times \mathbb{R}^{2}\right)$, and $0<\alpha, \beta \leq 1$. Let $y$ : $[a, b] \longrightarrow \mathbb{R}$ be a $\gamma$-differentiable function with $y(a)=y_{a}$, and $y(b)=y_{b} \in \mathbb{R}$. Let $y\left(\partial L / \partial \mathrm{D}_{\mathrm{h}, \mathrm{k}, \mathrm{l}}^{\alpha, \beta} y\right)$ be a differentiable function, and $\left(\partial L / \partial \mathrm{D}_{\mathrm{h}, \mathrm{k}, \mathrm{l}}^{\alpha, \beta} y\right)$ be $\gamma$-differentiable. If $y$ is an outer of $\mathfrak{J}$, then $y$ satisfies the following Euler-Lagrange

Solving for $\lambda$, it then follows Eq. (102). 
equation:

$$
\begin{aligned}
& \frac{\partial L\left(x, y(x), \mathrm{D}_{\mathrm{h}, \mathrm{k}, \mathrm{l}}^{\alpha, \beta} y(x)\right)}{\partial y} \\
& -\mathrm{D}_{\mathrm{h}, \mathrm{k}, \mathrm{l}}^{\alpha, \beta}\left(\frac{\partial L\left(x, y(x), \mathrm{D}_{\mathrm{h}, \mathrm{k}, \mathrm{l}}^{\alpha, \beta} y(x)\right)}{\partial \mathrm{D}_{\mathrm{h}, \mathrm{k}, \mathrm{l}}^{\alpha, \beta} y}\right)=0 .
\end{aligned}
$$

Proof. We define a family of functions:

$$
\bar{y}(x)=y(x)+\epsilon \eta(x),
$$

where $\epsilon$ is a constant and $\bar{y}(x)$ satisfies the same boundary conditions as $y . \eta(x)$ is an arbitrary $\gamma$-differentiable function, which satisfies the boundary conditions $\eta(a)=\eta(b)=0$.

Since $\mathfrak{J}=\mathfrak{J}(\epsilon)$, to make $\mathfrak{J}$ stationary

$$
\begin{gathered}
\left.\frac{d}{d \epsilon}\right|_{\epsilon=0} \int_{a}^{b} L\left(x, y(x), \mathrm{D}_{\mathrm{h}, \mathrm{k}, \mathrm{l}}^{\alpha, \beta} y(x)\right) \mathrm{d} \gamma(x)=0 \\
\left.\int_{a}^{b} \frac{\partial}{\partial \epsilon} L\left(t, y(x), \mathrm{D}_{\mathrm{h}, \mathrm{k}, \mathrm{l}}^{\alpha, \beta} y(x)\right)\right|_{\epsilon=0} \mathrm{~d} \gamma(x)=0 .
\end{gathered}
$$

Applying the fractional chain rule (Property 8),

$$
\begin{aligned}
& \left.\int_{a}^{b}\left[\frac{\partial L}{\partial \bar{y}} \frac{\partial \bar{y}}{\partial \epsilon}+\frac{\partial L}{\partial \mathrm{D}_{\mathrm{h}, \mathrm{k}, \mathrm{l}}^{\alpha, \beta} \bar{y}} \frac{\partial \mathrm{D}_{\mathrm{h}, \mathrm{k}, \mathrm{l}}^{\alpha, \beta}}{\partial \epsilon}\right]\right|_{\epsilon=0} \mathrm{~d} \gamma(x)=0 \\
& \left.\int_{a}^{b} \frac{\partial L}{\partial \bar{y}} \eta\right|_{\epsilon=0} \mathrm{~d} \gamma(x) \\
& +\left.\int_{a}^{b} \frac{\partial L}{\partial \mathrm{D}_{\mathrm{h}, \mathrm{k}, \mathrm{l}}^{\alpha, \beta} \bar{y}} \mathrm{D}_{\mathrm{h}, \mathrm{k}, \mathrm{l}}^{\alpha, \beta}\right|_{\epsilon=0} \mathrm{~d} \gamma(x)=0 .
\end{aligned}
$$

Integrating and applying the boundary conditions to $\eta(x)$ in the second integral,

$$
\left.\int_{a}^{b}\left[\frac{\partial L}{\partial \bar{y}}-\mathrm{D}_{\mathrm{h}, \mathrm{k}, \mathrm{l}}^{\alpha, \beta}\left(\frac{\partial L}{\partial \mathrm{D}_{\mathrm{h}, \mathrm{k}, \mathrm{l}}^{\alpha, \beta} \bar{y}}\right)\right] \eta(x)\right|_{\epsilon=0} \mathrm{~d} \gamma(x)=0 .
$$

Evaluating Eq. (111) at $\epsilon=0$ it then follows Eq. (105).

The fractional Sturm-Liouville eigenvalue problem (65. 66. 67) is equivalent to the following:

Finding the stationary function $y(x)$ of

$$
F[y]=\int_{a}^{b}\left(p\left(\mathrm{D}_{\mathrm{h}, \mathrm{k}, \mathrm{l}}^{\alpha, \beta} y\right)^{2}-q y^{2}\right) \mathrm{d} \gamma(x) .
$$

Subject to $G[y]=1$, where

$$
G[y]=\int_{a}^{b} w y^{2} \mathrm{~d} \gamma(x) .
$$

To find the stationary function of $F[y]$ subject to $G[y]=$ 1 , we first find the stationary value of $K[y]=F[y]-\lambda G[y]$, and then, eliminate $\lambda$ using $G[y]=1$.

$$
K[y]=\int_{a}^{b}\left[p\left(\mathrm{D}_{\mathrm{h}, \mathrm{k}, \mathrm{l}}^{\alpha, \beta} y\right)^{2}-q y^{2}-\lambda w y^{2}\right] \mathrm{d} \gamma(x) .
$$

Applying the new Euler-Lagrange equation, Eq. (105), to Eq. (114) and rearranging:

$$
\mathrm{D}_{\mathrm{h}, \mathrm{k}, \mathrm{l}}^{\alpha, \beta}\left(p \mathrm{D}_{\mathrm{h}, \mathrm{k}, \mathrm{l}}^{\alpha, \beta} y\right)+q y=-\lambda w y
$$

which is the Sturm-Liouville eigen-value problem defined in Eq. (65).

Multiplying (65) by $y$ and integrating by parts yields

$$
\begin{aligned}
\left.p y \mathrm{D}_{\mathrm{h}, \mathrm{k}, \mathrm{l}}^{\alpha, \beta} y\right|_{a} ^{b} & -\int_{a}^{b}\left(p\left(\mathrm{D}_{\mathrm{h}, \mathrm{k}, \mathrm{l}}^{\alpha, \beta} y\right)^{2}+q y^{2}\right) \mathrm{d} \gamma(x) \\
& =-\lambda \int_{a}^{b} w y^{2} \mathrm{~d} \gamma(x) .
\end{aligned}
$$

Since the boundary conditions are of Neumann type, py $\left.\mathrm{D}_{\mathrm{h}, \mathrm{k}, \mathrm{l}}^{\alpha, \beta} y\right|_{a} ^{b}=0$, thus

$$
\lambda \int_{a}^{b} w y^{2} \mathrm{~d} \gamma(x)=\int_{a}^{b}\left(p\left(\mathrm{D}_{\mathrm{h}, \mathrm{k}, \mathrm{l}}^{\alpha, \beta} y\right)^{2}+q y^{2}\right) \mathrm{d} \gamma(x)
$$

Applying constrain Eq. (113) to Eq. (117)

$$
\lambda=\int_{a}^{b}\left(p\left(\mathrm{D}_{\mathrm{h}, \mathrm{k}, \mathrm{l}}^{\alpha, \beta} y\right)^{2}+q y^{2}\right) \mathrm{d} \gamma(x)
$$

That is, $\lambda$ is determined by $F[y]$ in Eq. (112).

Now we present some results, which we shall use later when solving some examples of the Sturm-Liouville eigenvalue problem.

Lemma 5.9 Let $\alpha, \beta \in(0,1], h(x)>0, k(x)>0$ and $\mathrm{d} \gamma(x)=h^{-\beta}(x) k^{\alpha-1}(x) \mathrm{d} x$. Then,

$$
\begin{aligned}
\mathrm{D}_{\mathrm{h}, \mathrm{k}, \mathrm{l}}^{\alpha, \beta}\left(\sin \left(\int^{x} \mathrm{~d} \gamma(t)\right)\right) & =\cos \left(\int^{x} \mathrm{~d} \gamma(t)\right) \\
\mathrm{D}_{\mathrm{h}, \mathrm{k}, \mathrm{l}}^{\alpha, \beta}\left(\cos \left(\int^{x} \mathrm{~d} \gamma(t)\right)\right) & =-\sin \left(\int^{x} \mathrm{~d} \gamma(t)\right) \\
\mathrm{D}_{\mathrm{h}, \mathrm{k}, \mathrm{l}}^{\alpha, \beta} e^{\left(\int \mathrm{d} \gamma(t)\right)} & =e^{\left(\int^{x} \mathrm{~d} \gamma(t)\right)} .
\end{aligned}
$$


Proof.

$$
\begin{aligned}
\mathrm{D}_{\mathrm{h}, \mathrm{k}, \mathrm{l}}^{\alpha, \beta}\left(\sin \left(\int^{x} \mathrm{~d} \gamma(t)\right)\right) & =h^{-\beta}(x) k^{1-\alpha}(x) \\
\frac{d}{d x} \sin \left(\int^{x} \mathrm{~d} \gamma(t)\right) & =h^{-\beta}(x) k^{1-\alpha}(x)\left(h^{\beta}(x) k^{\alpha-1}(x)\right) \cos \left(\int^{x} \mathrm{~d} \gamma(t)\right) .
\end{aligned}
$$

The proof of Eq. (120) is smiliar to this one.

$$
\mathrm{D}_{\mathrm{h}, \mathrm{k}, \mathrm{l}}^{\alpha, \beta} e^{\left(\int^{x} \mathrm{~d} \gamma(t)\right)}=h^{-\beta}(x) k^{1-\alpha}(x) \frac{d}{d x} e^{\left(\int^{x} \mathrm{~d} \gamma(t)\right)}=h^{-\beta}(x) k^{1-\alpha}(x)\left(h^{\beta}(x) k^{\alpha-1}(x)\right) e^{\left(\int^{x} \mathrm{~d} \gamma(t)\right)} .
$$

\subsection{Examples}

(1) Example 1. We now use the New Rayleigh Quotient in two examples to obtain a lower estimate for the first eigenvalue. Setting $p=1, q=0, w=1, h(x)=x, k(x)=x^{2}$ and $y(x)=x^{\alpha \beta}-x^{2 \alpha \beta}$, which satisfies $y(0)=y(1)=0$, then from Eq. (102):

$$
\lambda_{1} \leq \frac{\int_{0}^{1}\left(\mathrm{D}_{\mathrm{h}, \mathrm{k}, \mathrm{l}}^{\alpha, \beta}\left[x^{\alpha \beta}-x^{2 \alpha \beta}\right]\right)^{2}\left(x^{2}\right)^{\alpha-1} x^{-\beta} \mathrm{d} x}{\int_{0}^{1}\left(x^{\alpha \beta}-x^{2 \alpha \beta}\right)^{2}\left(x^{2}\right)^{\alpha-1} x^{-\beta} \mathrm{d} x} \leq \frac{\alpha^{2} \beta^{2}\left(-\frac{4}{3 \alpha \beta-2 \alpha+\beta+1}+\frac{4}{4 \alpha \beta-2 \alpha+\beta+1}+\frac{1}{2 \alpha(\beta-1)+\beta+1}\right)}{\frac{1}{4 \alpha \beta+2 \alpha-\beta-1}+\frac{1}{-3 \alpha \beta-2 \alpha+\beta+1}+\frac{1}{(2 \alpha-1)(\beta+1)}} .
$$

If we set $\alpha=0.7$ and $\beta=0.2$

$$
\lambda_{1}=0.613988 .
$$

Applying the same conditions, except for $\beta$ and $h(x)$ and setting them $\beta=h(x)=1$, then performing the integration, we recover the result from Abdeljawad and Al-Refai [5]:

$$
\lambda_{1} \leq 10 \alpha^{2} .
$$

(2) Example 2. Now, we set $p=1, q=0, w=1, h(x)=x, k(x)=x^{2}$, and $y(x)=x^{1 / 3}-x^{1 / 5}$, which satisfies $y(0)=y(1)=0$ :

$$
\begin{aligned}
\lambda_{1} & \leq \frac{\int_{0}^{1}\left(\mathrm{D}_{\mathrm{h}, \mathrm{k}, \mathrm{l}}^{\alpha, \beta}\left[x^{\frac{1}{3}}-x^{\frac{1}{5}}\right]\right)^{2}\left(x^{2}\right)^{\alpha-1} x^{-\beta} \mathrm{d} x}{\int_{0}^{1}\left(x^{\frac{1}{3}}-x^{\frac{1}{5}}\right)^{2}\left(x^{2}\right)^{\alpha-1} x^{-\beta} \mathrm{d} x} \leq \frac{1}{8}(30 \alpha-15 \beta-7)(10 \alpha-5 \beta-3)(6 \alpha-3 \beta-1) \\
& \times\left(-\frac{2}{-30 \alpha+15 \beta+23}+\frac{1}{-50 \alpha+25 \beta+35}+\frac{1}{-18 \alpha+9 \beta+15}\right) .
\end{aligned}
$$

(3) Example 3. Solving the Eigenvalue Problem defined in (65) with $p=1, q=0, w=1$, with the aid of Lemma 5.9, we get the eigenfunctions:

$$
y=\sin \left(n \pi f(\alpha, \beta) \int^{x} \mathrm{~d} \gamma(x)\right) \text {, }
$$

with eigenvalues:

$$
\lambda=n^{2} \pi^{2} f^{2}(\alpha, \beta) .
$$

If we set $\alpha=0.7, \beta=0.2$ and $f(\alpha, \beta)=\beta\left(e^{-\alpha}+e^{-\beta}\right)$, we get:

$$
\lambda_{1}=0.682999 .
$$

Setting $f(\alpha, \beta)=\alpha$, for the first eigenvalue, we recover the result from Abdeljawad and Al-Refai:

$$
\lambda=\alpha^{2} \pi^{2} .
$$




\section{Conclusiones}

We introduced a new differential operator and explored its properties, and showed that we could recover both Khalil [1] and Katugampola's [2] definitions of the conformable fractional derivative, as well as the regular integer-order derivative. We introduced the inverse operator $\mathrm{I}_{\mathrm{h}, \mathrm{k}, \mathrm{l}}^{\alpha, \beta}$ and explored its properties, which allowed us to get an expression for Integration by parts analogous to the integer order expression. We also developed some operators, such as the anticommutator $\widehat{C}_{\gamma_{1}, \gamma_{2}}$ as well as a self-adjoint variant of it, $\widehat{A}_{2 \alpha, 2 \beta}$, which allowed us to recover the Sturm-Liouville operator in terms of integer order derivatives, by multiplying $\widehat{C}_{2 \alpha, 2 \beta}$ with an appropriate weight function which is the extra-term in our definition. Afterward we were able to show some examples of differential equations using this self-adjoint operator. We then considered a more general case by solving the SturmLiouville eigenvalue problem. We generalized the Rayleigh Quotient, the Lagrange identity, the Euler-Lagrange equation, and we showed that the eigenvalues are real and that the eigenfunctions are orthogonal. We then discussed some examples and compared the upper bound for the eigenvalues from the Rayleigh Quotient with the eigenvalues obtained by the eigenfunctions obtained by solving the Sturm-Liouville eigenvalue problem.

\section{Acknowledgments}

We appreciate the support provided by Universidad Iberoamericana Ciudad de México and DINVP.
1. R. Khalil, M. Al Horani, A. Yousef, and M. Sababheh, A new definition of fractional derivative. Journal of Computational and Applied Mathematics 264 (2014) 65. https://doi. org/10.1016/j.cam.2014.01.002

2. U. N. Katugampola, A new fractional derivative with classical properties, e-print. arXiv preprint arXiv:1410.6535 8 (2014), https://arxiv.org/abs/1410.6535v2

3. D. R. Anderson and D. J. Ulness, Properties of the Katugampola fractional derivative with potential application in quantum mechanics. Journal of Mathematical Physics, 56 (2015) 063502.https://doi.org/10.1063/1.4922018

4. R. Almeida, M. Guzowska, and T. Odzijewicz, A remark on local fractional calculus and ordinary derivatives. Open Mathematics, 14 (2016) 1122 https://doi.org/10.1515/ math-2016-0104.

5. T. Abdeljawad, On conformable fractional calculus. Journal of computational and Applied Mathematics, 279 (2015) 57. https://doi.org/10.1016/j.cam.2014.10.016

6. N. Benkhettou, S. Hassani, and D. F. Torres, A conformable fractional calculus on arbitrary time scales. Journal of King Saud University-Science, 28 (2016) 93. https://doi. org/10.1016/j.jksus.2015.05.003

7. A. Atangana, D. Baleanu, and A. Alsaedi, New properties of conformable derivative. Open Mathematics, 1 (2015) http: //hdl.handle.net/20.500.12416/2879.

8. T. Chiranjeevi, and R. K. Biswas, Closed-form solution of optimal control problem of a fractional order system. J. King Saud University-Science, 31 (2019) 1042. https://doi.org/ $10.1016 / j . j k s u s .2019 .02 .010$

9. M. Al-Refai, and T. Abdeljawad, Fundamental results of conformable Sturm-Liouville eigenvalue problems. Complexity, (2017), https://doi.org/10.1155/2017/3720471

10. D. Zhao, X. Pan, and M. Luo, A new framework for multivariate general conformable fractional calculus and potential applications. Physica A: Statistical Mechanics and its Applications, 510 (2018), 271. https://doi.org/10.1016/ j.physa.2018.06.070
11. M. J. Lazo, and D. F. Torres, Variational calculus with conformable fractional derivatives. IEEE/CAA Journal of Automatica Sinica, 4(2), (2016), 340-352, https://doi.org/ $10.1109 /$ JAS.2016.7510160.

12. M. Klimek, and O. P. Agrawal, Fractional Sturm-Liouville problem. Computers and Mathematics with Applications, 66(5), (2013), 795-812, https://doi.org/10.1016/j. camwa.2012.12.011

13. M. N. Alam, and X. Li, New soliton solutions to the nonlinear complex fractional Schrödinger equation and the conformable time-fractional Klein-Gordon equation with quadratic and cubic nonlinearity. Physica Scripta, 95 (2020) 045224, https://doi.org/10.1088/1402-4896/ab6e4e

14. F. S. Silva, D. M. Moreira, and M. A. Moret, Conformable Laplace transform of fractional differential equations. Axioms, 7 (2018) 55. https://doi.org/10.3390/ axioms 7030055

15. A. Younas, T. Abdeljawad, R. Batool, A. Zehra, and M. A. Alqudah, Linear conformable differential system and its controllability. Advances in Difference Equations, 2020 (2020) 1. https://doi.org/10.1186/ s13662-020-02899-0

16. F. Martínez, I. Martínez,M. K. Kaabar, R. Ortíz-Munuera, and S. Paredes, Note on the conformable fractional derivatives and integrals of complex-valued functions of a real variable. IAENG International Journal of Applied Mathematics, 50 (2020) 1. http: / / www. iaeng.org/IJAM/issues. v50/issue_3/IJAM_50_3_18.pdf.

17. Dazhi Zhao, Maokang Luo, General conformable fractional derivative and its physical interpretation, Calcolo, 54 (2017) 903 https://doi.org/10.1007/ s10092-017-0213-8

18. O. T. Birgani, Sumit Chandok, Nebojša Dedović, Stojan Radenović, A note on some recent results of the conformable derivative, Advances in the Theory of Nonlinear Analysis and its Applications 3 (2019) 11. https://doi.org/10.31197/ atnaa.482525 
19. A. Atangana, D. Baleanu, and A. Alsaedi, New properties of conformable derivative, Open Math 13 (2015) 889. http: //hdl.handle.net/20.500.12416/2879

20. S. Tuluce Demiray, New solutions of Biswas-Arshed equation with beta time derivative, Optik-International Journal for Light and Electron Optics 222 (2020) 165405. https:// doi.org/10.1016/j.ijleo.2020.165405
21. S. Tuluce Demiray, New Soliton Solutions of Optical Pulse Envelope E $(\mathrm{Z}, \tau)$ with Beta Time Derivative, Optik-International Journal for Light and Electron Optics 223 (2020) 1. https: //doi.org/10.1016/j.ijleo.2020.165453

22. Wolfram Research, Inc., Mathematica, Version 11.1, Champaign, IL (2017). 